\title{
Surface Decorated Mesoporous Silica Nanoparticles: A Promising and Emerging Tool for Cancer Targeting
}

\author{
Priya Virendrakumar Shah, Sadhana Rajput* \\ Faculty of Pharmacy, Maharaja Sayajirao University of Baroda, Centre of Relevance and Excellence in New Drug Delivery System, \\ Government of India, Vadodara, Gujarat, INDIA.
}

\begin{abstract}
Background: In nanotechnology, the most promising inorganic materials for cancer targeting are Mesoporous silica nanoparticles (MSNs). This review gives a state of the art description of current status and applications of surface functionalized MSNs in the field of cancer theranostics. It also gives a detailed description of surface decorated MSNs researched upon so far in various types of cancer and the benefits associated with these. Applications: Ease of surface functionalization also offers additional functionalities like cell recognition, absorption of specific biomolecules, improving cell interaction and cellular uptake that significantly modifies the in vitro and in vivo behavior of the drug. Therefore, they have proved to be effective in gene delivery and multi drug resistance cancer treatment. Though, their biocompatibility still remains debatable. Biodegradation of MSNs is quite simple and it is safely excreted through kidneys via silanoic acid formation. Moreover, the USFDA has already approved the silica under the 'GRAS' category which has further made MSNs a thrust area for research in cancer theranostics. Summary: This review summarizes the journey of multifunctional MSNs beginning with its origin, mechanism of formation of mesoporous structure with surface functionalization to till date and recent advances in the arena of cancer targeting.
\end{abstract}

Key words: Mesoporous silica nanoparticles, Cancer targeting, Surface modification, Theranostics, Biomedical Application, Biodegradation.

\section{INTRODUCTION}

Cancer is second leading causes of death amongst all the lethal diseases prevalent. It is estimated globally that nearly 1 in 6 deaths is because of cancer. ${ }^{1}$ Which puts a tremendous pressure on the researchers to find a cure for the same. Every day new advances are being made towards cancer treatment, by synthesizing new moieties or by researching new molecular mechanisms. Current drug molecules available for cancer treatment include peptides, steroid molecules and oligonucleotides. Most of them are hydrophobic in nature and hence, possessing low solubility and bioavailability. Therefore, the desired therapeutic dose will not reach to the target and requires a higher dose for therapeutic effectiveness. This may damage healthy cells and tissues, leading to severe side effects like, severe hair falls resulting in baldness, acute vomiting and nausea, low blood cell counts making patients more susceptible towards developing infection or anaemia. $^{2}$

Since last couple of decades, research breakthroughs have been made in outlining pharmaceutical medications for different disorders. This has significantly propelled the learning of physico-chemical properties and cellular uptake mechanisms and thereby generating effective therapeutic strategies. In chemotherapy, the present treatment strategies basically rely on the utilization of ordinary cytotoxic medications which have adverse effects and restricted efficacy. Numerous studies ascribe this to the inefficiency of anti-cancer drugs in reaching the target site. To tame this obstacle, a target specific nano drug delivery system is employed
Submission Date: 30-07-2018; Revision Date: 19-12-2018; Accepted Date: 14-04-2019

DOI: 10.5530/ijper.53.3.72 Correspondence: Prof. Sadhana Rajput Faculty of Pharmacy, Kalabhavan Campus, The Maharaja Sayajirao University of Baroda, Centre of Relevance and Excellence in New Drug Delivery System, Government of India, Vadodara390002, Gujarat, INDIA. Phone: +91-026-52434187 E-mail: sjrajput@gmail.com

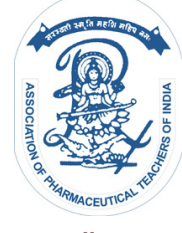

www.ijper.org 
for effective delivery of drug to the targeted area, thus minimizing its adverse effects. Furthermore, nano sized drug delivery system can passively targeted to cancer cells by Enhanced permeability and retention (EPR) effect. ${ }^{3,4} \mathrm{An}$ ideal drug delivery system should encourage the intracellular accumulation of drug in the targeted cells and maintain drug concentration to its effective level. This is possible by two ways viz., (a) by designing a formulation that gives controlled or sustained release or (b) by concentrating the drug to its target i.e. tumor cell. The research thrust these days is on designing a targeted drug delivery system that will deliver a therapeutic dose to the cancerous cell and ultimately reduce its reach to healthy cells thereby decreasing their side effects. ${ }^{5}$ Targeting concept is now in focus and vast researches are involved to formulate a nano system, including liposomes, ${ }^{6-8}$ solid lipid nanoparticles, ${ }^{9}$ Self-micro emulsifying drug delivery system (SMEDDS). ${ }^{10}$ Mesoporous silica nanoparticle (MSNs) is one of the nano carriers, which are in focus for targeting the tumor cells. ${ }^{10,11}$

MSNs based targeted delivery offers a promising tool for increasing the intra tumoral concentration of anticancer drugs and limiting its toxic effects to normal cells as they bind to cell membrane receptors which are over expressed in cancer cells only. This has led to a great research interest towards targeting the drug loaded MSNs into tumor cells. The Unique properties of MSNs like flexible surface, porosity, shape, size, surface functionalization etc collectively decide mechanism of action, their interaction with living body components such as cells, tissues and biomolecules and release pattern of formulation in biological systems, with considerable increment in therapeutic ability. Together, all these have a direct impact on safety, biodistribution and theranostic potential. ${ }^{12}$ Moreover, theranostic application of MSNs is also in the limelight where, the name theranostic itself suggests dual application of MSNs in therapeutic and diagnosis filed. This dual characteristic is solely attributed to its flexible surface that can easily be modified to achieve the desired objective. ${ }^{13}$ MSNs with particle size ranging from 50-200 $\mathrm{nm}$ have been studied as nanocarriers for the delivery of small drug molecules and antigen and it has been proved that MSNs are having high drug loading capacity. ${ }^{14-18}$ Furthermore, tumor targeting has been specifically accomplished either by functionalization of nano-carriers having selective and specific interaction with tumor-over expressed receptors $^{19-21}$ or by designing stimuli-responsive nano therapeutics or combination of both. ${ }^{22,23}$ It has been proven that tumor cells are more acidic as compared to healthy cells and this concept can be utilized for targeting of MSNs by coating their surface with different $\mathrm{pH}$ responsive material. E.g. $\mathrm{pH}$ responsive microspheres having a core of $\mathrm{Fe}_{3} \mathrm{O}_{4}$ nanoparticle, a bilayer of mesoporous silica and a shell of crosslinked poly methacrylic acid (PMAA), were successfully synthesized via distillation precipitation polymerization. ${ }^{24}$ The temperature difference between normal and cancerous cell is also an another approach used for targeting. ${ }^{25}$ Redox, ${ }^{26}$ light $^{27}$ and enzyme ${ }^{28}$ are some of the emerging strategies for the same. Moreover, MSNs also possess unique features like biocompatibility, high drug loading and entrapment efficiency, stability on storage, cell specificity and site directing ability. ${ }^{5,29}$ Despite of all these applications, they are yet to be explored and commercialized. A general review of the recent advances in cancer targeting using MSNs is presented here.

\section{Mesoporous Silica Nanoparticles}

Mesoporous materials are in the limelight due to their potential applications in catalysis, adsorption, ecology, nanotechnology, chemical and biological separation, chromatography, photonic and electronic device preparation and medical uses. Since last decade mesoporous silica has shown tremendous potential in drug delivery applications. ${ }^{30}$ MSNs possess unique features like large surface areas, pore volume and pore size. Different types of carriers available are shown in Table $1 .{ }^{31-33}$ Amongst various nano drug delivery systems, MSNs are emerging as a promising drug carrier, which can perform all the aforementioned functions at the same time. After the discovery of highly ordered mesoporous silica material MCM-41 (Mobil crystalline of materials) by the mobile corporation in 1992, noteworthy research efforts have been in progress to accomplish control over the characteristics of mesoporous silica especially pore size and morphology. Lately, various types of mesoporous materials have been discovered e.g. MSU, SBA, HMS, OMS, TUD and MCF were developed with typical pore size and particle shapes. ${ }^{34,35}$

The unique identities possessed by MSNs as mentioned earlier helps in encapsulating a variety of therapeutic agents and also in targeting them to cancerous cells. Notably, the fabrication of MSNs is simple, scalable, cost-effective and controllable. ${ }^{36}$ MSNs have also been widely used as a controlled drug release and delivery carriers, ${ }^{37-39}$ biosensors, ${ }^{40}$ bio-markers, ${ }^{41}$ enzyme supporters, ${ }^{42,43}$ etc. Usually, MSNs are utilized as a drug delivery system because of their unique 2D hexagonal framework, uniform molecular sizes, controllable molecular morphology with the tendency of modifying inner and outer surface. ${ }^{44}$ Apart from having such unique qualities, MSNs have a couple of additional advantages like high thermal and chemical stability. Also, the ease of 
functionalization makes them perfect for use as a support for adsorption and catalysis. ${ }^{45}$ Conclusively, it could be said that MSNs are nanomaterials having characteristics of both silica as well as mesoporous materials and can be functionalize MSNs surface by silica chemistry and also integrate the desired material within the silica matrix. $^{46}$

\section{Design and Preparation of MSNs}

\section{Principle Behind Formation of MSNs}

The general mechanism of MSNs synthesis includes condensation of silica precursors governed by selfassembled liquid-crystal arrays of surfactants. Thorough studies state two proposed mechanisms for the formation of MSNs. The first synthesis procedure was proposed by Mobile corporation scientists in 1992 named as a Liquid crystal templating (LCT) mechanism. ${ }^{47}$ As per this proposed mechanism surfactants have a structure directing property which encourages the formation of the mesophase. Hence, the mesoporous structure formation depends on the hydrocarbon chain length of the surfactant tail group and it has been proved that the increment in the surfactant concentration may initiate aggregation of spherical into cylindrical or rod-like micelles. In other words, this mechanism involves a condensation of silicate ions around the preformed hexagonal surfactant array (Sol-gel formation). The final step involves exclusion of surfactant to get a highly ordered hexagonal pattern as shown in Figure $1 .{ }^{48}$ However, according to another strategy, surfactant will form a micellar structure which interacts with silica and bi to tri-layered silica will form surrounding the micellar assembly. Spontaneously these species will self-organize to form a well-ordered hexagonal structure. ${ }^{49}$

\section{Various Elements Influencing Drug Loading}

Application of mesoporous material as a drug carrier is being comprehensively studied in pharmaceutical field

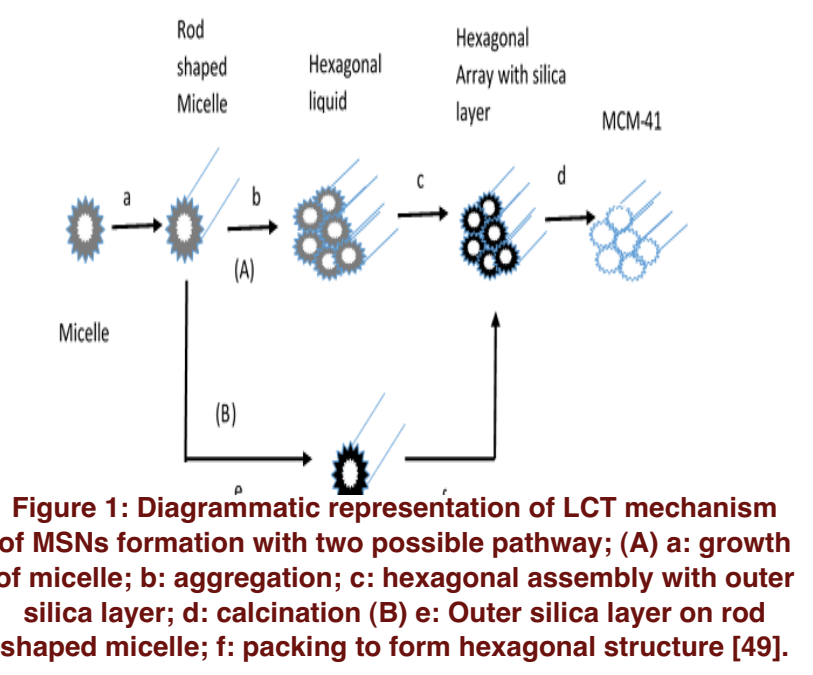

to develop targeted drug delivery systems. Loading of the drug in MSNs is the most critical and key portion contributing to success of formulation which ultimately depends on the selection of an appropriate loading condition. There are several factors that affect the encapsulation of the drug in the MSNs like loading method, pore size, pore volume, surface area, surface chemistry, effect of solvent, temperature and time of stirring, etc as described further. ${ }^{30,50}$

Loading methodology plays a vital role in drug encapsulation within nanoparticles. The type of loading method used, affects the extent of drug being loaded into the mesopores, their fitting into the pores and distribution in the carrier. The various methods used for drug loading in MSNs are an immersion method in which the MSNs are immersed in concentrated drug solution. The drug molecules fill the pore of nanoparticles through capillary action followed by drug diffusion in MSNs and adsorption on the pore wall. The drug loaded nanoparticles are collected by filtration. Ibuprofen loaded MCM41 was obtained by ensuing this method. ${ }^{51}$ In Incipient wetness impregnation method a highly concentrated drug solution is used for loading of the drug whose concentration is close to its solubility. Once the solvent gets evaporated, the chances of recrystallization of residual drug become high in this method. This approach has been applied for encapsulation of various drugs such as Aceclofenac, ${ }^{52}$ Carvedilol, ${ }^{53}$ Ezetimibe,${ }^{54}$ Fenofibrate, ${ }^{55}$ Indomethacin. ${ }^{56,57}$ Melt method is a loading method where a physical mixture of MSNs carrier and drug is prepared and ignited above the melting point of the drug. However, there is a high chance of degradation of drug as the mixture is heated above the melting point. ${ }^{50}$ In Rotavapor method instead of using normal or vacuum filtration procedure, rota evaporator is used for removal of the solvent. A concentrated drug solution is prepared, followed by addition of carrier and stirring for a predefined time at a particular stirring speed, subjected to evaporation in rota evaporator. Indomethacin drug has been successfully loaded by this method. ${ }^{56}$ The same approach has been applied to Atazanivir. ${ }^{58}$ In a Fluidized bed method, the solvent is evaporated by spraying and heating the suspension using the fluidized bed machine. Indomethacin has been prepared by this method using MCM-41 as a carrier. It was concluded that degree of loading was higher in rotavapor and fluidized bed method compared to the conventional impregnation method..$^{56}$ Apart from the loading method, the type of solvent, degree of stirring, temperature of stirring can also affect the drug loading inside the pores. However, from the various factors affecting drug loading, type 
of loading method employed is a key factor having an active impact on the degree of success of drug loading.

\section{Surface Functionalization of MSNs}

\section{Surface Functionalization of MSNs Through Mussel- Inspired Chemistry}

Mussel chemistry is a very important tool useful in surface functionalization of nanocomposites. The facileness and versatility of mussle inspired chemistry make it an attractive concept to follow. ${ }^{59,60}$ Mussel adhere strongly in wet conditions to wood and are underwater specialist due to different mussel foot proteins containing 3,4-dihydroxy-L-phenylalanine (DOPA) and lysine amino acids secreted during adhesive formation in the adhesive plaque of mussel byssus. ${ }^{61}$ Mussel inspired polydopamine coating has garnered a significant attention due to simplicity, material independency in deposition, favorable cell interaction and strong reactivity for secondary functionalization. ${ }^{6}$ Since PDA contain both catechol and amine groups it is used as a one-step facile method for surface coating in nanoparticles useful in material science, biomedical, biology and environmental fields. ${ }^{60,63-65} \mathrm{PDA}$ is an emerging soft matter and a crucial component of mussel inspired chemistry. ${ }^{66}$ Wei et al. synthesized novel fluorescent organic nanoprobes for biological imaging applications based on self-polymerization of dopamine and polyethyleneimine. ${ }^{67} \mathrm{~A}$ similar principle is applied to inorganic silica nanoparticles.

Biosilification between PDA and silicic acid is utilized. PDA blocks are selectively incorporated into silica framework by controlled addition of Dopamine. The PDA-MSN hybrid nanocomposites have proved to be advantageous exhibiting a high drug loading, sustained drug release and enhanced anticancer efficacy. ${ }^{68}$ They can also serve as useful nanocatalyst and antimicrobial agent. ${ }^{69}$ PDA-MSNs are responsive to $\mathrm{pH}$ stimulus and hence can act as an effective anticancer agent. The PDA coating works as an effective gatekeeper for time bound $\mathrm{pH}$ sensitive controlled release of drug. ${ }^{69}$ Polydopamine and peptide decorated MSN has been researched upon as potential targeted drug delivery system for bladder cancer therapy. ${ }^{70}$

\section{Pore Gating}

This system involves unifying of bulky molecular moieties like proteins and peptides or incorporation of metals like iron (Superparamagnetic iron oxide nanoparticles; Fe-NP) and gold (A gold nanoparticle; Au-NP). Giri S, et al. formulated the mesoporous silica nanorods with iron oxide (Fe-NP) cap, possessing a redox responsive cleavable disulphide linker which upon cleavage releases the cargo. ${ }^{71}$ One more pore gating MSNs has been designed by researchers A Schlossbauer, et al. which deals with the strategy of melting of DNA linkers by MSNs decorated with temperature programmable molecular valve system comprising avidin caps. ${ }^{72}$

\section{External surface modification of MSNs}

Outer layer of MSNs carrier is feasible for surface modification and provides an opportunity to prepare tunable MSNs. This external surface decoration may affect the release profile and may possess targeting efficiency. ${ }^{73}$

Two basic mechanisms exist for grafting of functional groups on the surface of carrier viz., physical and chemical. These also depend on the solubility of the drug. Physical adsorption is commonly seen in external grafting of MSNs. Hydrogen bonding, electrostatic and hydrophobic interactions are the representative forms of the physical adsorption. In case of non-functionalized MSNs, where plenty of $\mathrm{OH}$ groups are available on the surface, hydrogen bond becomes a representative of physical adsorption, but once the surface is functionalized latter two types of interaction will represent physical adsorption. ${ }^{50}$

Two main strategies are applied for surface modification viz., co-condensation and post-synthetic grafting method. In the former technique, functionalizing ligand is administered during the synthesis of bare MSNs, whereas in the latter case, functionalizing moiety is incorporated once the unmodified MSNs carrier is synthesized. $^{74}$

\section{Co-condensation process}

The process of co-condensation involves direct functionalization of MSNs' outer surfaces during synthesis only. This strategy modifies mesoporous silicate surface by sol-gel chemistry between tetra-alkoxysilane and one or more organo-alkoxysilanes with $\mathrm{Si}-\mathrm{C}$ bonds and this will synthesize hybrid inorganic-organic mesoporous silicates. This method is also applicable for synthesis of MSNs for imaging purposes. E.g. use of co-condensation method for incorporation of two different $\mathrm{Gd}^{3+}$ complexes at very high loading $(15.5 \%-28.8 \% \mathrm{w} / \mathrm{w})$ and this synthesized, MCM-41 carrier was successfully characterized by SEM, TEM, TGA, PXRD, DCP. General steps involved in synthesis of MSNs (Addition of silica source i.e. TEOS, surfactant, water etc., autoclaving and calcination) are displayed in Figure 2.75,76

\section{Post synthesis strategy}

This process involves the surface modification after synthesis, i.e. post synthesis functionalization method. It involves modification of the surface of MSNs usually after removal of surfactant. Here, the Si-OH group 
which is present on the surface of MSNs acts as an anchoring moiety for functionalization. It is mostly carried out by silylation process. Grafting can be done on internal as well as external surface. The detailed pictorial diagram is depicted in Figure 3. ${ }^{76}$

The co-condensation method is preferred as found in most of the literature due to more uniformity. e.g. Surface of MCM-41 was functionalized by vinyl group using both the above-mentioned techniques. Lim and Stein have compared the relative distribution of surface groups based on powder X-ray diffraction (XRD), $\mathrm{X}$-ray photoelectron spectroscopy (XPS) and bromination kinetics data. Results showed a wider distribution of vinyl groups on the surface of MCM-41 prepared by direct co-condensation method, whereas for those prepared by the post grafting method, the results showed a lack of uniformity with a large proportion of vinyl groups on the external surface of the crystallites or inside channels but lesser number near the channel openings. ${ }^{77}$ In products obtained from a direct cocondensation reaction, the vinyl groups appeared to be more uniformly distributed throughout the channels. A number of functional groups have been trying to set the functionalized MSNs which could be used as drug car-

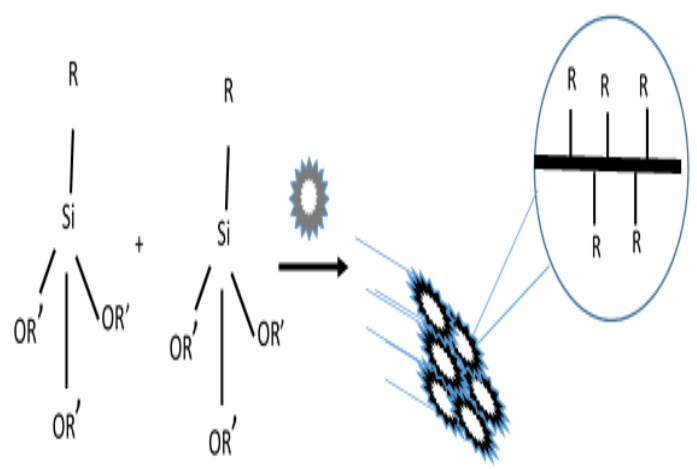

Figure 2: Basic steps involved in MSNs synthesis. ${ }^{64,65}$

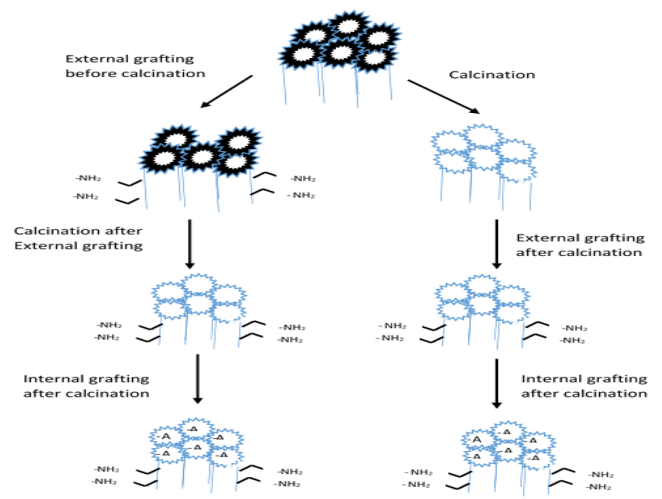

Figure 3: Two different strategy for functionalization of carrier. ${ }^{65}$ riers. E.g. Outer decoration of MSNs with disulphide linked polymer to achieve the redox responsive release of drug has been done by author Liu $\mathrm{R}$, et al. ${ }^{78} \mathrm{~A}$ variety of functional group or moieties can be attached on the surface of MSNs by either of the above mentioned techniques as listed in the Table 2. ${ }^{11,79-90}$

Backfilling strategy is another approach used for MSNs fabrication. It is a simple approach to introduce active molecules into the empty pores of mesostructured silica wherein, these empty pores are exposed to the solution or vapour of active molecules and are allowed to diffuse. However, this strategy does not involve chemical modification of the material and hence is a less widely used method. ${ }^{5}$

\section{Tunable Mesoporous Silica Nanoparticles}

Surface functionalization results in the alteration of MSNs feature like pore size, pore diameter, pore volume, surface area, etc. The pore structure can be modified prior to the surface modification by thermally annealing in an inert nitrogen atmosphere. This treatment reduces the overall pore volume, since it causes pore coalescence, which may melt pores together. Particle size and particle shape are unique properties of MSNs and solely represent the release profile of the drug.

\section{Particle Size}

\section{Mesoporous silica microspheres}

Mesoporous silica has size ranging from nano to micro size, i.e. mesoporous silica nanomaterials and mesoporous silica micromaterials respectively. Well established method for synthesis of monodisperse non-porous silica particles are Stober method. This method involves the use of TEOS (A silica source), alkyltrialkoxysilane (Porosity generating molecule), ethanol (Co-solvent), water and aqueous ammonia (Catalyst). ${ }^{91}$ Unger and Stucky are the first group to synthesize micrometer sized mesoporous silica spheres. ${ }^{92,93}$ It was concluded that by controlling the ratio of water, ammonia, ethanol and other reagents one can get micron to submicron sized mesoporous silica sphere with varying porosity and surface area.

\section{Mesoporous silica nanospheres}

A well-known mesoporous silica nanocarrier is MCM41, which usually involves the use of TEOS as a silica source. Often fumed silica is used as a silica source, cationic surfactant CTAB or CTAC is added as a structure directing agent and water is the used as a solvent. These are a very commonly used ingredients for mesoporous silica nanosphere synthesis by various researchers. But the proportions of these materials are varied depend- 
ing upon the synthesis scheme which ultimately makes a difference in surface area, pore diameter and pore volume, even though utilizing the same ingredients (Tuning of surface area and pore volume). The MSNs are exposed to facile endocytosis in both plant and animal cell without exhibiting much cytotoxicity, this is attributed to their tunable particle size ranging from 50 to 300 $\mathrm{nm}$. A typical MSNs have an average particle diameter, surface area, pore size and pore volume around $100 \mathrm{~nm}$, $>900 \mathrm{~m}^{2} / \mathrm{g}, 2 \mathrm{~nm}$ and $>0.9 \mathrm{~cm}^{3} / \mathrm{g}$ respectively with the hexagonal structure. This can be altered by modifying the time required for hydrothermal treatment, molar composition of ingredients used and duration of calcination. ${ }^{38}$

\section{Particle Shape}

MSNs can have various shapes like spheres, rod, tube etc. This again depends on ratio and type of ingredient taken during synthesis. E.g. incorporation of double concentration of TEOS, $\mathrm{NaOH}$ and CTAB gives tubular MSNs rather regular spherical MSNs with the same pore size and structure. By increasing the APTMS/ TEOS ratio from $1.28 \%$ to $12.8 \mathrm{~mol} \%$, shape simultaneously changes from kidney bean rod to sphere. ${ }^{94}$ Replacing APTMS with AAPTMS and AEPTMS gives twisted columns and micrometer size mesoporous silica spheres. Using 1-octadecyl-3-methylimidazolium as a surfactant template leads to the generation of a helical structure instead of parallel channels. ${ }^{95}$

\section{Recent Development of AIE-Active Materials for Biomedical Applications}

Synthesis of Fluorescent polymeric nanoparticles (FPNs) with AIE emission has been widely researched upon. An effective and facile method for their synthesis is microwave-assisted Kabachnik-Fields method which gives AIE active FPNs possessing high water dispersity, intensive fluorescence and low cytotoxicity which enhances their applicability in biomedical field apart from being a facile, fast and atom economical strategy. ${ }^{96}$ The AIE phenomenon was first reported in 2001 by Tang and co-workers. ${ }^{97,98}$ FPNs based on conventional organic dyes suffer from Aggregation caused quenching (ACQ) and effect and hence exhibit reduced fluorescence intensity. Whereas organic dyes with AIE characteristics show a weak fluorescence in dilute solution and strong one in aggregated state. This can easily conquer the drawbacks of ACQ effects. Also, majority of organic dyes suffer from biocompatibility and stability issues which needs to be addressed. Certain strategies useful in achieving this are non-covalent self-assembly, covalent conjugation, controlled living polymerization, formation of dynamic bonds and anhydride ring opening polymerization. ${ }^{99-102}$ Other synthesis procedures are catalyst free azide-alkyne click reaction, ${ }^{103}$ thionyl click reaction, ${ }^{104}$ dynamic phenyl borate bond formation, ${ }^{105}$ multicomponent reaction (One-pot strategy). ${ }^{106,107}$ Most prominent applications of AIE induced FPNs is in bioimaging ${ }^{96,108}$ with certain modifications proving to be a boon like salicylic acid-poly-PEG-co-vinyl aniline which provide fluorescent nanoparticles having excellent biocompatibility, enhanced cellular uptake and superior photo bleaching resistance behaviour. ${ }^{109}$ Similar advances have been made in preparation of luminescent MSNs as discussed further.

\section{Fabrication of Luminescent MSNs}

Intrinsic/label-free Luminescent mesoporous silica nanoparticles (L-MSNs) have proved to be highly useful in biomedical field and have various applications in drug delivery. MRI contrast agent, cell sorting and cell labelling. ${ }^{110}$ Uniform pore size, multi functionality and low cytotoxicity are the major reasons for popularity of MSNs over other nanocarriers in biomedical applications. However, synthesis of luminescent or magnetic MSNs need to be properly monitored and optimized since irregular pore structures lead to diminished efficacy in biological systems. Label-free approach has garnered lot of attention and is a facile way of Luminescent-MSN synthesis. Jin et al. ${ }^{111}$ found that calcination of MSNs at $400^{\circ} \mathrm{C}$ gave mesoporous organosilicon NPs with strong fluorescence of ultimate photo and chemical stability. It was reported that the origin of luminescence were carbon dots generated from calcination and not due to the silica matrix defects as contrary to the traditional belief. Furthermore, it was also observed that the calcination did not affect the drug loading and biomolecule conjugating properties of the MSNs. The novel calcination induced fluorescence could contribute to MSNs synthesis for use in drug delivery, nanoparticle coating and immunofluorescence imaging. Other methods include labelling of silica particles with a fluorophore like Organic dye molecule loaded by chemical conjugation or physical adsorption or a NP fluorophore like Quantum dot (QDs) ${ }^{112}$ or up conversion NPs. ${ }^{13,114}$ However, there are several drawbacks of this methods when compared with label-free technique; like time consuming, expensive, high toxicity, increased particle size and dye leakage. ${ }^{115,116}$ Another approach of bottom up self-assembly followed by post calcination was used in synthesizing oxygen deficient luminescent MSNs for drug loading/delivering and cell imaging. Triethoxy silane was employed as a silicon source to create oxygen vacancies by dehydrogenation between terminal groups 
during post-calcination. Electrochemical etching of silicon wafer in HF based electrolyte solutions followed by ultrasonic fabrication or mechanical milling and subsequent centrifugation or filtration causes poor size and morphology control on MSN pores along with low yield due to unwanted micro particles also obtained. ${ }^{117,118}$ Jain and group ${ }^{119}$ also synthesized highly stable L-MSNs by optimizing APTS and TES ratio (Stober process followed by calcination) and concluded that formation of carbon dots was responsible for Luminescence as confirmed by fluorescence anisotropy measurements. Another example of L-MSN synthesis includes their application in photodynamic therapy. A photosensitizer zinc pthalocyanine was incorporated into MSN and excited by NIR laser through which activated nanocrystals could convert NIR light to visible light which in turn activated photosensitizer to release reactive singlet oxygen species to kill cancer cells. ${ }^{114}$

\section{Biomedical Applications of MSNs}

MSNs serve as a magic bullet for theragnostic medicine and in biomedical applications. ${ }^{74}$ Mechanized MSNs functionalized with nanomachines proffer enumerable advantages as nanotheranostic agents. Some of them include possessing a higher surface area to volume ratio which leads to ease of surface functionalization to fit any desired need related to targeting and theranostics. Thus, ease of surface functionalization makes it easier to attach various targeting moieties using a simple surface functionalization process. This ease of surface decoration or ligand attachment is not prevalent on large scale in case of other nanocarriers and polymeric micelles. Polymeric luminescent nanomaterials also possess multifunctional ability and good photostability. ${ }^{120}$ However, MSNs apart from all these aspects also possess zero premature leakage. A major issue associated with Fluorescent inorganic nanoparticles (FINs) is the problem of in vivo biodegradability associated with them attributed to their accumulation in reticuloendothelial system. On the other hand, Silica comes under the category of GRAS (Generally regarded as safe) for human use and also used as an additive in tablet manufacturing. Inside body also it is converted to a non-toxic silicic acid and excreted mostly through urine. Although, a new class of Fluorescent organic nanoparticles (FONs) provide excellent biocompatibility and good dispersibility. FONs with aggregation induced emissions hold a good potential in various bio-imaging applications. ${ }^{121,122} \mathrm{~A}$ broader excitation wavelength is desirable for biomedical applications. ${ }^{100}$ Although, Various organic dyes possess rapid labelling, non-toxicity and easier clearance, they are vulnerable in biological environments and exhibit aggregation caused quenching (ACQ). ${ }^{123}$ Fluorescent silica nanoparticles offer advantages like higher quantum yield and better fluorescence stability as compared to small organic fluorescent molecules. ${ }^{124} \mathrm{PhE}-\mathrm{PEG}$ FONs were reported to possess excellent biocompatibility, water dispersibility and fluorescence. ${ }^{125}$ Semiconductor quantum dots and metal clusters possess several disadvantages like intrinsic cytotoxicity and difficulty in surface functionalization. ${ }^{126} \mathrm{In}$ both the aforementioned aspects MSNs hold an upper hand. Nanodiamonds are another form of delivery system widely being investigated upon. Te cyclodextrin based nanocomposites possess greater water dispersibility, high drug loading and controlled drug release. ${ }^{105}$ However, comparatively MSNs also offer tunable pore size which can easily control site and time of cargo release; apart from possessing high payload capacity.

\section{Targeting by MSNs}

MSNs can be altered by providing good end capping, precise targeting and stimuli triggered drug release to fulfil the aim of efficient drug loading with minimum side effects. Previously, organic molecules,${ }^{10}$ inorganic nanoparticles ${ }^{127}$ and molecular switches ${ }^{128}$ etc, were employed to block the mesopores of MSNs for preventing drug leakage. MSNs can be targeted to cancer cells implementing receptor-based targeting approach or stimuli responsive targeting strategy. Various receptor binding ligand used so far involved lactose, ${ }^{129}$ folic acid, ${ }^{128}$ DNA aptamer, ${ }^{27}$ peptides ${ }^{130}$ and phenylboronic acid $^{131}$ that follows the former approach of targeting. Whereas, letter strategy requires an efficient stimulus to trigger drug release inside the tumor cells. An external stimuli can be divided into physical stimuli like light, ${ }^{27}$ magnetic field ${ }^{132}$ etc and biological stimuli in the tumor microenvironment like redox ${ }^{128} \mathrm{pH}^{111}$ enzyme ${ }^{133,134}$ etc. Details of both the approach are summarized below.

\section{Stimuli responsive MSNs targeting}

MSNs have been explored in depth for stimuli-based cargo delivery. Various stimuli like $\mathrm{pH}$, redox, enzyme, magnetic field and temperature are widely used to stimulate MSNs prepared. This strategy is highly useful in addressing the issue related to off target toxicity in cancer therapy. ${ }^{135}$ The controlled release is obtained at three levels (1) using gatekeepers by pore sealing with particle or molecular gates, (2) surface coating of particle and (3) coupling of cargo onto the internal walls of pores. ${ }^{136}$ They further depend on two types of responses first is a conformational change of the pore sealing agent and the second is exposure to stimuli which can lead to the cleaving of bonds. Frequent method used in latter case 
is cleavage of disulphide bond as a part of redox stimuli-based cargo delivery system. A summary of recent stimuli responsive mesoporous systems having an application in cancer is enumerated in Table $3 .{ }^{137-146}$

\section{$\mathrm{pH}$}

The $\mathrm{pH}$ responsive behaviour is most researched upon and accepted worldwide as a tumor specific drug delivery of anticancer drugs. The basic fundamental involved here is the fact that tumor micro-environment has an acidic $\mathrm{pH}$ than normal tissues. Thus, designing a delivery system which can release drug at an acidic $\mathrm{pH}$ can be highly beneficial for targeted delivery of anticancer drugs. The difference in $\mathrm{pH}$ can be credited to the high proliferative rate of cancer cells, leading to an increased lactic acid production and enhanced efflux of protons present within the cell. ${ }^{147}$ Recently Nasab et al. ${ }^{148}$ have synthesized a $\mathrm{pH}$ based mesoporous drug delivery system for curcumin using natural polymer chitosan for capping. There was an appreciable increase in the solubility of curcumin and remarkable improvement in its therapeutic efficacy as tested against U87MG glioblastoma cancer cell line. There was also encouraging results when $\mathrm{IC}_{50}$ values of free curcumin and capped MSNs were calculated with almost three times enhancement in cytotoxicity at same $\mathrm{IC}_{50}$ dose. Similarly, Kienzle and co-workers designed a $\mathrm{pH}$ stimuli responsive dendritic mesoporous network for TNF-Alpha. The stimuli system consisted of a block copolymer gate system synced with charged hyper branched polyethylenimine and nonionic hydrophilic PEG for TNF-Alpha encapsulation and delivery into various cancer cell lines and dendritic cells. There was a reduction in $\mathrm{EC}_{50}$ for prepared MSNs by more than two times and showed stability in the media up to five days. ${ }^{149}$ Further, MSNs have also been employed in lymphoma treatment. E.g. Zhou et al. have developed an intracellular $\mathrm{pH}$ responsive conjugated MSN system for rituximab targeted delivery to lymphoma B cells. Avidin-biodin bridging approach was undertaken and the results showed little premature release at physiological $\mathrm{pH}$ and enhance release in microtumor environment leading to enhanced therapeutic value and diminished off-target toxicity. ${ }^{150}$

\section{Redox}

The fundamental involved here is that in the human body, glutathione-GSH/ GSH disulphide is one of the major redox couplets which play a pivotal role in minimizing the damage caused due to Reactive oxygen species (ROS). ${ }^{151}$ The most important fact to consider here is that in tumor cells, the GSH concentration is elevated 100 to 1000 times more and this is exploited in designing of redox stimuli based delivery system. ${ }^{26,151}$ GSH acts as an effective, reducing agent for the cleavage of disulphide bonds. Chen et al. ${ }^{152}$ have reported the synthesis of transferrin gated MSNs for Redox based Doxorubicin (DOX) release. The idea was successful as there was a burst release when exposed to GSH, due to the cleavage of disulphide bonds in MSNs. Transferrin served a dual purpose of both capping and targeting agents. Site specific delivery was achieved with this biocompatible carrier. A major breakthrough was achieved by Zhao and group when they synthesized MSNs for co delivery of Si-RNA and DOX through MSNs. Where, Si-RNA was joined to the core via disulphide linkage and interestingly played a gatekeeper role. There was an enhanced accumulation of DOX in the cancer cells and prepared MSNs could knock down expression of target proteins. Thus, they exhibited a wide tumor targeting potential. ${ }^{109}$

\section{Magnetic field}

Magnetic MSNs have been widely explored for diagnosis and treatment of cancer. Although, a strong magnetic field may not always be an economically feasible option, still they are being researched upon due to the positive results and encouraging outcomes they have so far given in cancer theranostics. ${ }^{153}$ They require minimum one exogenous stimulus for responding to magnetic field. MSNs as MRI contrast agents have been more intensively studied. They are also in the limelight due to their proved biocompatibility. ${ }^{154} \mathrm{Li}$ et al. designed a magnetic MSN system for co delivering of DOX and VEGF Si RNA for MR imaging and cervical cancer targeting. This included a Magnetic mucus penetrating nanoparticles (MMPP) by employing the ultrasound emulsification method and Superparamagnetic iron oxide nanoparticles (SPIONS) encapsulated into a blend of PLGA and PLGA-PEG polymers. Under magnetic field explosion the penetration speed of prepared NPs was found to be increased three fold than in the absence of magnetic field. ${ }^{155}$

It is widely known that the therapeutic efficacy of Photodynamic therapy (PDT) is limited by hypoxia associated with cancer due to the fact that PDT is an oxygen concentration dependent process. Kim et al. ${ }^{156}$ designed a biocompatible manganese ferrite anchored MSNs to overcome hypoxia thereby enhancing the therapy of PDT. This can be attributed to the continuous oxygen evolving property of $\mathrm{Mn}-\mathrm{Fe}_{2} \mathrm{O}_{4}$ nanoparticles via Fenton reaction. Additionally, modified MSNs also exhibited a T2 contrast effect in MRI imaging having a benefit of in vivo tracing of administered MSNs. Thus, they exhibited a huge potential in theranostic therapy 
of cancer. Tian and group synthesised magnetic MSNs coated with a thermoresponsive polymer i.e. P(NIPAMco-MAA). The magnetic supermagnetic nanoparticles were loaded with DOX and exhibited both $\mathrm{pH}$ and temperature responsive behaviour. They had higher efficacy in killing cancerous HeLa cells. It was also proved that magnetic MSNs are capable of higher internalization in cancer cells. ${ }^{157}$

\section{Enzyme}

Enzyme responsive cargo delivery system has been developed in the mesoporous silica framework. The MSNs have been employed as theranostic agents for cancer therapy. Hu et al. had developed an enzyme Matrix metallo proteinase II (MMP-II) based MSNs for tumor targeted drug delivery and imaging. MMP-II activated fluorescent imaging peptides served as diagnostic probes as well nanovalves which blocked the pores based on enzyme responsiveness. MMP-2 overexpression in tumor tissue also triggered the drug release effectively. ${ }^{158}$ Recently Chunlin et al. employed a mussel mimetic enzyme responsive coating on MSN. They reported a facile strategy for polylysine-dopamine film coating via self-polymerization of dopamine derivative dopamine-lysine under basic conditions. Once the formulation reaches cancer cell, DOX was released via enzyme responsive degradation of peptide bonds. ${ }^{151}$ Tukappa and co-workers reported polyglutamic acid gated enzyme based drug delivery in metastatic breast cancer treatment. ${ }^{159}$ This system was developed for efficient co-delivery of Rhodamine and DOX. The cargo delivery occurred in pronase triggered manner. More than 90 percent of SK-BR-3 cancer cells was killed in the 100-ppm concentration and the developed NPs were proved to be non-toxic as well.

\section{Light}

Light is another stimuli used to trigger a selectively controlled release in cancer cells. Lu J et al. has engineered a camptothecin loaded and light activated MSNs having azobenzene impellers, which were photoactivated at specific wavelengths to trigger the impellers and lead to liberation of drug under light excitation. ${ }^{160}$

\section{Temperature}

Temperature is also applied as external stimuli to direct cargo release inside the cancer cells. It is mainly achieved by attaching thermosensitive poly ( $\mathrm{N}$-isopropyl acrylamide) derivative. ${ }^{25,161}$ Whereas, another research group has utilized octadecyltrimethoxysilane and paraffins to prepare thermo sensitive MSN where an increase in temperature above the melting point of paraffin resulted in cargo release. ${ }^{162}$

\section{Dual stimuli}

Dual or multiple stimuli responsive MSNs have also been developed and tested successfully for effectiveness and biocompatibility. As the name suggests here more than one stimuli are at work for cargo release, mostly employed for theranostic purposes. Recently, Wang et al. fluorescent carbon-dot gated multifunctional MSNs for redox and enzyme (Dual) responsive for the purpose of achieving controlled drug delivery and real time bioimaging. ${ }^{163}$ Furthermore, Zhu and group have prepared dual thermo and magnetic responsive nanoparticles. ${ }^{164}$

\section{Receptor Based MSN Targeting}

Various receptors have been explored so far to study targeting efficiency of MSNs. E.g. Khosravian and coworkers have engineered a docetaxel loaded folic acid or methionine functionalized MSNs. From the in vitro cell line, in vivo biodistribution study and ex vivo fluorescence imaging data, it was concluded that folate capped MSNs displayed significant tumor targeting efficiency with respect to methionine modified MSNs. As cancer cell displayed an overgrowth of folate receptor, a folate capped MSNs displayed relatively high affinity toward tumor cells. ${ }^{165}$ Similarly, Yu et al. had synthesised doxorubicin incorporated and hyaluronic acid modified MSN to target CD44 receptors overexpressed tumor cells. The success of drug concentration inside the tumor cells was confirmed by performing cellular uptake studies by confocal microscopic experiment and flow cytometric analysis. ${ }^{89}$ Goel S. et al. had formulated Vascular endothelial growth factor receptor (VEGFR) targeted MSNs with the goal of treating VEGF overexpressed tumor cells. Herein, the researcher had prepared NOTA-MSN-VEGF ${ }_{121}$ nanoparticle using PEG linkers. Where, VEGF ${ }_{121}$ was used as a VEGFR binding ligand. The in vitro, in vivo and ex vivo finding supported a successful synthesis of tumor targeted nanoparticles. ${ }^{166}$

\section{Toxicity, Biocompatibility and Biodegradation of MSNs}

Although having a tremendous application in the pharmaceutical world, the biocompatibility of MSNs is still debatable and it is essential to discuss about their biocompatibility and safety profile. Numerous parameters have direct influence on the safety profile of MSN which covers surface properties, particle size, charge, shape etc. At cellular level, MSNs can interact with the biological system through many of the mechanisms like mitochondrial dysfunction, glutathione depletion, membrane peroxidation and DNA damage etc. Research also says that the pristine MSNs are having less hemocompatibility as compared to surface deco- 
rated MSNs. Furthermore, apart from having targeting properties, the surface decoration exhibits reduced undesirable interaction with the internal organelles. ${ }^{167}$ Moreover, $\mathrm{Lu}$ et al. concluded $50 \mathrm{~nm}$ particle size is the optimal size for the drug delivery by MSNs with respect to $30 \mathrm{~nm}$ or even $200 \mathrm{~nm} .{ }^{168}$ Whereas, He et al. demonstrated that as the size of MSNs increases, the excretion from urine also increases correspondingly. Further, they also claimed that smaller size MSNs i.e. $190 \mathrm{~nm}$ and $420 \mathrm{~nm}$ size MSNs are more cytotoxic with respect to bigger sized MSNs i.e. $1220 \mathrm{~nm} .{ }^{169}$ Further the smaller nanoparticles are having more haemolytic effect as compared to bigger sized nanoparticles. ${ }^{170}$ Overall, there are dual thoughts for biocompatible nature of MSNs and it further requires an in depth exploration. Their biodegradation is debatable. Chen et al. have demonstrated a detailed degradation of MSNs in human embryo kidney $293 \mathrm{~T}$ cells by measuring the silica content in culture medium and performing depth TEM analysis. They claimed, upon degradation of MSNs into the cells, the size and dispersibility of cells were unchanged and hence the toxicity due to accumulation of silica aggregates in the tissues would be reduced. ${ }^{171}$ Further, Zhai et al. inferred that the degradation of MSNs took place in cytoplasm initially followed by in lysosomes later on. Moreover, the culture of MSNs with human endothelial cells experiments revealed higher accumulation of MSNs in the culture media i.e. outside the cell supports the claim that MSNs are not having cell toxicity. ${ }^{172}$ Additionally, research also says that the MSNs are converted into silanoic acid inside the body, which is excreted through kidney majorly. Further, the study also says that the silica particles mainly converts into its bioavailable form i.e. monomeric orthosilicic acid, which is essential for bone and connective tissue hemostasis. ${ }^{167}$

\section{Current Application of MSNs in Cancer Treatment}

MSNs are being explored for wide disease categories till now. Applications of MSNs in varied cancers are reported by many research groups. Few of them are explained briefly here.

\section{Breast Cancer}

(A) MY Hanafi-Bojd and group have synthesized an epirubicin loaded and MUC1 aptamer conjugated MSNs. The formulated nanoparticles were characterized thoroughly and were investigated for in vitro cellular uptake study on human breast carcinoma cells i.e. MCF-7 cells. Cytotoxicity results displayed significant breast targeted efficiency for aptamer attached MSNs with respect to unmodified MSNs and thus the formulation could improve the efficacy and possessed least adverse effect. ${ }^{173}$
(B) Ma'mani L, et al. has synthesized curcumin loaded and guanidine functionalized pegylated MSNs for breast cancer therapy. The surface of MSNs was modified by the isocyanate group with the aid of 3-(triethoxysilyl) propyl isocyanate (TESPIC) reagent under appropriate conditions. This surface modified MSNs were pegylated using PEG (MW 600Da) and finally with guanidine. To this multifunctionalized MSNs, curcumin was successfully loaded. Surface diameter was decreased from 834.61 (bare MSNs) to 234.6 (Curcumin loaded guidine+PEG coated MSNs) $\mathrm{m}^{2} / \mathrm{g}$ with a particle size range of $60-70$ $\mathrm{nm}$. The success of this functionalization was confirmed by various analytical techniques like DSC, FT-IR, BET, etc. In vitro release study showed higher and tailormade drug release in acidic $\mathrm{pH}(5.4 \mathrm{pH})$ compared to neutral $\mathrm{pH}$ (7.4). The results themselves depicts that curcumin showed $\mathrm{pH}$ responsive controlled release i.e. more release in an acidic environment (Tumor cells). The cell internalization study was successfully done by using the human breast carcinoma MCF-7 cell line. This showed a successful targeted delivery of curcumin to breast cancer cell. ${ }^{174}$

(C) A Second strategy of targeting involves enzyme responsive MSNs preparation. Briefly, it involves synthesis of MSNs as per the literature method available. Synthesised MSNs have been functionalized with carboxylic group and peptide sequence and finally with human serum albumin (HSA) having particle size of $80 \mathrm{~nm}$. To this functionalized carrier i.e. HSA labelled MSNs, DOX was successfully loaded. Cell apoptotic assay on HepG2 cells displayed a promising curative effect in breast targeted enzyme responsive delivery by $\mathrm{MSNs}^{28}$

(D) Song $\mathrm{H}$, et al. has synthesized folic acid-chitosan conjugated nanoparticles for improving tumor targeted drug delivery. Synthesized MSNs showed average particle size of $38 \pm 2 \mathrm{~nm}$ having positive zeta potential values. Further, this MSNs showed a biphasic release pattern of DOX from folate-chitosan coated MSNs compared to chitosan coated MSNs. Fluorescent images showed higher internalization of folate modified MSNs by folate receptor positive SMMC-7221 cell line compared with folate receptor negative MCF-7 cell line and this can be used as a promising tool for targeting a nanoparticles to cancerous cell. ${ }^{175}$

\section{Lung cancer}

It is the highest cause of death in patients suffering from cancer. It has been proven from the literature available that MSNs can also be used successfully for lung cancer targeting.

(A) Wang et al. have formulated paclitaxel loaded coreshell MSNs as a lung targeted nanoparticles. To investigate the targeted efficiency of synthesised nanoparticles, 
time dependent cellular uptake study and the apoptosis study were carried out on human A549 cells and results demonstrated the targeting potential poorly soluble drug for treatment of lung cancer. ${ }^{176}$

(B) Gao Y, et al. has synthesized albumin coated MSNs for the purpose of lung targeted controlled release of paclitaxel. In vitro cytotoxicity study was performed on lung cancer cell line A549 for formulated MSNs and results showed a significant anti-proliferative activity of albumin coated MSNs in lung cancer treatment. ${ }^{177}$

(C) Sundarraj S, et al. has synthesized EGFR antibody conjugated MSNs nanoparticles for lung targeted delivery. The mechanism involved here was receptor mediated endocytosis of antibody coated MSNs. Ligand receptor mediated recognition of EGFRab-SN pyrrolidine-2 nanoparticles attributed to higher cellular internalization through lung tumor cells, which was confirmed using human lung epithelial cell line L-132. ${ }^{178}$

\section{Prostate Cancer}

After lung cancer the second highest cause of cancer mortality in male is prostate cancer. Mannose 6 phosphate receptor is the main target of drug binding to prostate cancer. A carboxyl analogue (Mannose 6 carboxylate) of mannose 6 phosphate has been synthesized and this synthesized mannose 6 carboxylate was coated on MSNs following amination. TEM data revealed pore diameter of 15-25 nm. Further characterization was done by zeta potential and XRD analysis. In vitro cell line cytotoxicity study was performed on $\mathrm{LNCaP}$ prostate specific cell and results showed significant accumulation of MSNs inside the prostate cells. ${ }^{179}$

\section{Colorectal Cancer}

(A) Xie et al. has synthesized an aptamer coated and DOX encapsulated MSNs for treatment of epithelial cell adhesion molecule (EpCAM) overexpressed colon cancer. The targeting efficiency was performed on EpCAM over-expressing SW620 colon cancer cells and the aptamer coated MSNs exhibited significant inhibiting effect on the overexpressed EpCAM SW620 cells. ${ }^{180}$ (B) Here the strategy involves the development of eudragit fabricated hollow MSNs for targeted delivery. In the pharma field eudragit is widely used in enteric coated formulation, especially for $\mathrm{pH}$ responsive release i.e. to release a drug above $\mathrm{pH}$ 7. Eudragit was fabricated during synthesis of carrier (MCM-41) i.e. co-condensation method of surface functionalization having a particle size of $120 \mathrm{~nm}$. 5-flouro uracil has been loaded successfully into this carrier. In vitro, in vivo and cell cytotoxicity studies revealed a colon targeted release of 5-FU from the formulation. MSNs exhibited high drug loading with sustained and targeted drug delivery to the colon. ${ }^{181}$
(C) Tan L, et al. has developed glucose and $\mathrm{pH}$ responsive MSNs which selectively release the drug in an acidic environment. MSNs that are fabricated with Poly acrylic acid (PAA) brush on the surface with the aid of t-butyl acrylate. This PAA layer was again modified with glucosamine to obtain P(AA-AGA) and finally it was modified by adding 4,4-(ethylenedicarbamoyl)-phenylboronic acid (EPBA), showing the particle size of about 100 $\mathrm{nm}$. This will form boronate esters which will be disassociated in the presence of glucose and in an acidic environment. Here, the rate of release can be altered by changing $\mathrm{pH}$ or concentration of glucose in the surrounding environment. This combination showed a higher possibility of release of drug in mild acidic environment i.e. $\mathrm{pH} 6.0 .^{182}$

\section{Hepatic Cancer}

(A) Wang Z. et al. had formulated lactose mediated MSNs for delivery of platinum to liver cells by active targeting approach. The in vitro cell viability and cellular uptake study along with in vivo biodistribution study revealed prominence liver cell targeting efficiency of lactose coated MSNs. Further, the designed formulations displayed enhanced circulation time along with selective concentration inside the liver cells. ${ }^{183}$

(B) Quan G, et al. has synthesised lactosaminated mesoporous silica nanomaterials for asialoglycoprotein receptor (ASGPR) targeted delivery of docetaxel to hepatoma cells. MSNs carrier was synthesised as per Zink's report. Synthesised nanoparticles were functionalized with amino group by aminopropyltriethoxysilane reagent (APTES) and finally an attachment of lactose group was carried out with the aid of sodium cyanoborohydride solution. The calculated surface area was $1012 \mathrm{~m}^{2} / \mathrm{g}$ with the average pore size was $3.7 \mathrm{~nm}$ from the BET result with an approximate diameter of 100 $\mathrm{nm}$. It was found that Lactosaminated MSNs (LacMSNs) selectively bind with ASGPR in hepatic cells and showed a high hepatic targeting efficiency. Lac-MSNs were selectively endocytosed by ASGPR-positive hepatoma cell line, HepG2 and SMMC7721 cell line compare to ASGPR-negative NIH 3T3 cells. This was further confirmed by flow cytometry and confocal microscopic studies. ${ }^{11}$

\section{Pancreatic cancer}

One of the challenging category of cancer to treat is pancreatic cancer. Liu X, et al. have synthesized irinotecan loaded lipid bilayer coated MSNs having a particle size of $75-80 \mathrm{~nm}$, which lowered premature drug release with enhanced stability of formulating MSNs. The effectiveness of the formulation toward pancreatic tumor cells was studied on tumor specific orthotopic Kras-derived pancreatic ductal adenocarcinoma model 
and the engineered formulation was compared with liposomes. ${ }^{184}$

\section{Ovarian Cancer}

\section{The Ovarian Cancer Cell has also Been Targeted Successfully using MSNs}

(A) Guo and co-worker have formulated MSNs for active targeting of ovarian cancer. The research group has synthesized hollow MSNs loaded with DOX and NVP an inhibitor of insulin like growth factor receptor (IGF-1R). An IGF pathway was selected herein as a target as it is associated with the progression of ovarian cancer. The results displayed an active targeting of HMSN-COOH-DOX fluorescence NVP along with blocking the IGF pathway by NVP. Thus, the prepared MSNs were selectively targeted ovarian cancer cells. ${ }^{140}$

(B) Tumor endothelial marker 1 (TEM1)/endosialin (Ab-/scFv)-conjugated nanoparticles were synthesized by Zhang $\mathrm{Y}$, et al. Cellular uptake study and intracellular distribution of bevacizumab loaded and antibody conjugated MSNs showed promising results in human ovarian cancer cells i.e. OVCAR-5 cells. Thus, the significant accumulation of nanoparticles within the cells concluded the successful formation of ovary targeted nanoparticles. ${ }^{185}$

Apart from having a vital application in targeted nano technology, MSNs are having other multiple applications. E.g. A novel use of MSNs to overcome multidrug resistance (MDR) is now in the limelight. MDR is the major obstacle to be encountered during treatment of cancer. Co-administration of the MDR reversal agent along with disease treatment moiety improves the behaviour of MSNs toward MDR. For an instance, Lejiao jia, have formulated a multifunctional MSNs to overcome MDR of MCF-7/ADR cells by co-administering paclitaxel with tetrandrine, a MDR reversal agent to solve above problem regarding MDR. ${ }^{186}$ Likewise, Huan Meng et al. has formulated multifunctional MSN having the drug and the Si RNA combination to overcome MDR especially observed in breast cancer. The aim was to target the p-glycoprotein drug exporter following high throughput screening in MCF-/MDR. ${ }^{187}$ Moreover, MSNs are also recently been utilized for diagnostic purposes. MSNs are widely being researched upon for theranostic applications. Theranostic also termed as "theragnostic" is a term describing both therapeutic and diagnostic applications. MSNs are widely being researched upon for theranostic applications. Theranostic also termed as "theragnostic" is a term describing both therapeutic and diagnostic applications. Cheng et al. ${ }^{188}$ employed novel tri-functionalized MSNs for imaging, targeting and therapy of cancer. This all in one approach was found to be very effective, with this theranostic platform exhibiting minimal collateral damage, high therapeutic effect and maximum targeting specificity. The MSNs were sequentially functionalized with contrast agents that enable traceable imaging of particle targeting, drug payload and biomolecular ligands for therapeutic effect and targeted delivery of particle respectively. The entire system consisted of palladium-porphyrin based photosensitizer for PDT therapy and cRGDyK peptides for targeting of $\alpha_{\mathrm{v}} \beta_{3}$ integrins overexpressed in cancer cells. Each and every aspect of theranostic application was optimized successfully for imaging/targeting//therapy purposes. The latest theragnostic MSNs synthesized and their applications are summarized in Table $4{ }^{86,179,189-197}$ A schematic diagram of multifunctional MSNs is portrayed in Figure 4.

\section{Present Scenario}

Being a firm platform for satisfying multi purposes, still the field of MSNs has remained untouched and require to be explored. Cornell dots (C-dots form of MSNs) a USFDA approved product is in stage clinical trial. It is engineered for fluorescence imaging purpose and utilized for lymph node mapping in cancer. It includes cyclic RGD peptides as a targeting moiety, a polymer layer and NIR fluorescent dye labelled internal silica core. Silica has already obtained a tag of Generally regarded as safe (GRAS) but the same question arises for MSNs and it is to be answered. A first trial in human in 5 patients indicated a favourable pharmacokinetics and safety profile, creating opportunities for further trials in future. Silica listed under the GRAS category by USFDA. So far, it is already in use for human consumption as an excipient for tablets and food additive. Presently, silica nanoparticles are already in use in available commercial products like Nanoceuticals ${ }^{\mathrm{TM}}$ chocolate slim shake and Lancome ${ }^{\mathbb{R}}$ Renergie microlift, a cosmetic product. ${ }^{198}$ However, a major breakthrough

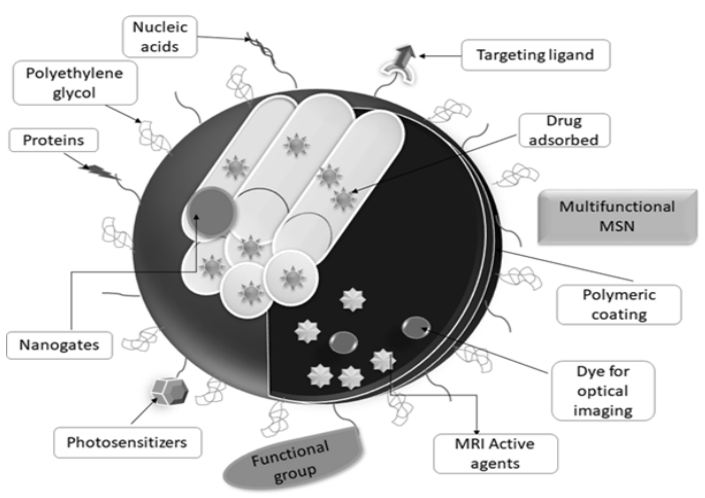

Figure 4: Multifunctional MSNs. 
was achieved when USFDA approved for the first time human trials for Cornell dots, a fluorescent core shell silica nanoparticles to be used as biological markers for diagnosis in metastatic melanoma patients. ${ }^{199}$

\section{CONCLUSION}

Of all the drug delivery platforms available MSNs do hold a special position due to the various advantages associated with them. Some of them being ease of surface functionalization, zero premature leakage and tunable pore size. The targeting efficiency of MSNs has been widely researched upon with numerous positive outcomes reported. In spite of the special attributes of MSNs a wide research needs to be done to determine their biosafety in human clinical trials. Luminescent MSNs and theranostic applications are the recent advances and relatively newer trends still in naive stage. However, they serve as the most suitable candidates for potential biomedical applications. They provide an ideal platform for drug and diagnostic agent delivery and are a valuable asset in bright future of biomedicine and nano delivery systems.

\section{ACKNOWLEDGEMENT}

Author would like to acknowledge the Maharaja Sayajirao University of Baroda for providing an excellent platform and facilities for the study.

\section{CONFLICT OF INTEREST}

The authors declare no conflict of interests.

\section{ABBREVIATIONS}

5-FU: 5-Flouro Uracil; AAPTMS: N-(2-Aminoethyl)3-Aminopropyltrimethoxysilane; AEPTMS: 3-[2-(2-Aminoethylamino)Ethylamino]Propyltrimethoxy-Silane; APTES: Aminopropyltriethoxysilane; APTES: 3-Aminopropyl Triethoxysilane; ASGPR: Asialoglycoprotein Receptor; CPTES: 3-Cyanopropyltriethoxysilane; CTAB: Cetyltrimethylammonium Bromide; CTAC: Cetyltrimethylammonium Chloride; DOX: Doxorubicin; EGFR: Epidermal Growth Factor Receptor; EPBA: 4,4-(Ethylenedicarbaamoyl)-Phenylboronic Acid; EpCAM: Epithelial Cell Adhesion Molecule; EPR: Enhanced Permeability And Retention; GRAS: Generally Regarded As Safe; HAS: Human Serum Albumin; IGF-1R: Insulin Like Growth Factor Receptor; LCT: Liquid Crystal Templating; MCM: Mobile Composition Of Matter; MDR: Multidrug Resistance; MMP-II: Metallo Proteinase II; MMPP:
Magnetic Mucus Penetrating Nanoparticles; MPS: Mononuclear Phagocytic System; MRI: Magnetic Resonance Imaging; MSNs: Mesoporous Silica Nanoparticles; MSU: Michigan State University; MTD: Maximum Tolerated Dose; PAA: Poly Acrylic Acid; PDT: Photodynamic Therapy; PEG: Polyethylene Glycol; PMAA: Poly Methacrylic Acid; PXRD: Powder X-Ray Diffraction; ROS: Reactive Oxygen Species; SBA: University Of California At Santa Barbara; SEM: Scanning Electron Microscopy; Si-RNA: Small Interfering Ribonucleic Acid; SMEDDS: Self-Micro Emulsifying Drug Delivery System; SPIONS: Superparamagnetic Iron Oxide Nanoparticles; TEM: Transmission Electron Microscopy; TEM1: Tumor Endothelial Marker 1; TESPIC: 3-(Triethoxysilyl) Propyl Isocyanate; TGA: Thermo Gravimetric Analysis; TUD: Technischeuniversiteit Delf; USFDA: United State Food And Drug Administration; VEGFR: Vascular Endothelial Growth Factor Receptor; XPS: X-Ray Photoelectron Spectroscopy; XPS: X-Ray Photoelectron Spectroscopy; XRD: $\mathrm{X}$-Ray Diffraction.

\section{REFERENCES}

1. http://www.who.int/mediacentre/factsheets/fs297/en/.

2. Gottesman MM, Fojo T, Bates SE. Multidrug resistance in cancer: role of ATP-dependent transporters. Nature Reviews Cancer. 2002;2(1):48-58.

3. Deng Z, Yan F, Jin Q, Li F, Wu J, Liu X, et al. Reversal of multidrug resistance phenotype in human breast cancer cells using doxorubicin-liposomemicrobubble complexes assisted by ultrasound. Journal of Controlled Release. 2014;174:109-16.

4. Oerlemans C, Bult W, Bos M, Storm G, Nijsen JFW, Hennink WE. Polymeric micelles in anticancer therapy: targeting, imaging and triggered release. Pharmaceutical Research. 2010;27(12):2569-89.

5. Bharti C, Nagaich U, Pal AK, Gulati N. Mesoporous silica nanoparticles in target drug delivery system: a review. International Journal of Pharmaceutical Investigation. 2015;5(3):124.

6. Sapra P, Allen T. Ligand-targeted liposomal anticancer drugs. Progress in Lipid Research. 2003;42(5):439-62.

7. Gabizon A, Shmeeda H, Horowitz AT, Zalipsky S. Tumor cell targeting of liposome-entrapped drugs with phospholipid-anchored folic acid-PEG conjugates. Advanced Drug Delivery Reviews. 2004;56(8):1177-92.

8. Deshpande PP, Biswas S, Torchilin VP. Current trends in the use of liposomes for tumor targeting. Nanomedicine. 2013;8(9):1509-28.

9. Xu Z, Chen L, Gu W, Gao Y, Lin L, Zhang Z, et al. The performance of docetaxel-loaded solid lipid nanoparticles targeted to hepatocellular carcinoma. Biomaterials. 2009;30(2):226-32.

10. Pan L, He Q, Liu J, Chen Y, Ma M, Zhang L, et al. Nuclear-targeted drug delivery of TAT peptide-conjugated monodisperse mesoporous silica nanoparticles. Journal of the American Chemical Society. 2012;134(13):5722-5.

11. Quan G, Pan X, Wang Z, Wu Q, Li G, Dian L, et al. Lactosaminated mesoporous silica nanoparticles for asialoglycoprotein receptor targeted anticancer drug delivery. Journal of Nanobiotechnology. 2015;13(1):7.

12. Mamaeva V, Sahlgren C, Lindén M. Mesoporous silica nanoparticles in medicine-Recent advances. Advanced Drug Delivery Reviews. 2013;65(5):689-702.

13. Chen NT, Cheng SH, Souris JS, Chen CT, Mou CY, Lo LW. Theranostic applications of mesoporous silica nanoparticles and their organic/inorganic hybrids. Journal of Materials Chemistry B. 2013;1(25):3128-35. 
14. Rao KS, El-Hami K, Kodaki T, Matsushige K, Makino K. A novel method for synthesis of silica nanoparticles. Journal of Colloid and Interface Science. 2005;289(1):125-31.

15. Stöber W, Fink A, Bohn E. Controlled growth of monodisperse silica spheres in the micron size range. Journal of Colloid and Interface Science. 1968;26(1):62-9.

16. Van HA, Jansen JW, Vrij A. Preparation and characterization of spherical monodisperse silica dispersions in nonaqueous solvents. Journal of Colloid and Interface Science. 1981;81(2):354-68.

17. Bogush G, Zukoski C. Studies of the kinetics of the precipitation of uniform silica particles through the hydrolysis and condensation of silicon alkoxides. Journal of Colloid and Interface Science. 1991;142(1):1-18.

18. Brinker CJ, Clark DE, Ulrich DR. Better ceramics through chemistry: symposium held February 1984 in Albuquerque, New Me xico, USA: NorthHolland. 1984.

19. Rosenholm JM, Meinander A, Peuhu E, Niemi R, Eriksson JE, Sahlgren C, et al. Targeting of porous hybrid silica nanoparticles to cancer cells. ACS Nano. 2008;3(1):197-206.

20. Ferris DP, Lu J, Gothard C, Yanes R, Thomas CR, Olsen JC, et al. Synthesis of Biomolecule-Modified Mesoporous Silica Nanoparticles for Targeted Hydrophobic Drug Delivery to Cancer Cells. Small. 2011;7(13):1816-26.

21. Knežević NŽ, Durand JO. Targeted treatment of cancer with nanotherapeutics based on mesoporous silica nanoparticles. Chem Plus Chem. 2015;80(1):2636.

22. Wang Y, Shim MS, Levinson NS, Sung HW, Xia Y. Stimuli-Responsive Materials for Controlled Release of Theranostic Agents. Advanced Functional Materials. 2014;24(27):4206-20.

23. Knežević NŽ, Trewyn BG, Lin VSY. Functionalized mesoporous silica nanoparticle-based visible light responsive controlled release delivery system. Chemical Communications. 2011;47(10):2817-9.

24. Wen H, Guo J, Chang B, Yang W. pH-responsive composite microspheres based on magnetic mesoporous silica nanoparticle for drug delivery. European Journal of Pharmaceutics and Biopharmaceutics. 2013;84(1):91-8.

25. Fu Q, Rama Rao G, Ward TL, Lu Y, Lopez GP. Thermoresponsive transport through ordered mesoporous silica/PNIPAAm copolymer membranes and microspheres. Langmuir. 2007;23(1):170-4.

26. Schafer FQ, Buettner GR. Redox environment of the cell as viewed through the redox state of the glutathione disulfide/glutathione couple. Free Radical Biology and Medicine. 2001;30(11):1191-212.

27. Yang X, Liu X, Liu Z, Pu F, Ren J, Qu X. Near-Infrared Light-Triggered, Targeted Drug Delivery to Cancer Cells by Aptamer Gated Nanovehicles. Advanced Materials. 2012;24(21):2890-5.

28. Liu J, Zhang B, Luo Z, Ding X, Li J, Dai L, et al. Enzyme responsive mesoporous silica nanoparticles for targeted tumor therapy in vitro and in vivo. Nanoscale. 2015;7(8):3614-26.

29. Tarn D, Ashley CE, Xue M, Carnes EC, Zink JI, Brinker CJ. Mesoporous silica nanoparticle nanocarriers: biofunctionality and biocompatibility. Accounts of chemical research. 2013;46(3):792-801.

30. Vadia N, Rajput S. Mesoporous material, MCM-41: a new drug carrier. Asian Journal of Pharmaceutical and Clinical Research. 2011;4(2):44-53.

31. Andersson J, Rosenholm J, Areva S, Lindén M. Influences of material characteristics on ibuprofen drug loading and release profiles from ordered micro-and mesoporous silica matrices. Chemistry of Materials. 2004;16(21):4160-7.

32. Davidson A. Modifying the walls of mesoporous silicas prepared by supramolecular-templating. Current Opinion in Colloid and Interface Science. 2002;7(1):92-106

33. Jansen J, Shan Z, Marchese L, Zhou W, Puil N, Maschmeyer T. A new templating method for three-dimensional mesopore networks. Chemical Communications. 2001(8):713-4.

34. Trewyn BG, Slowing II, Giri S, Chen HT, Lin VSY. Synthesis and functionalization of a mesoporous silica nanoparticle based on the solgel process and applications in controlled release. Accounts of Chemical Research. 2007;40(9):846-53.

35. Vallet-Regi M, Ramila A, Del RR, Pérez-Pariente J. A new property of MCM41: drug delivery system. Chemistry of Materials. 2001;13(2):308-11.
36. Tang F, Li L, Chen D. Mesoporous silica nanoparticles: synthesis, biocompatibility and drug delivery. Advanced Materials. 2012;24(12):150434

37. He Q, Shi J. Mesoporous silica nanoparticle based nano drug delivery systems: synthesis, controlled drug release and delivery, pharmacokinetics and biocompatibility. Journal of Materials Chemistry. 2011;21(16):5845-55.

38. Slowing II, Vivero-Escoto JL, Wu CW, Lin VSY. Mesoporous silica nanoparticles as controlled release drug delivery and gene transfection carriers. Advanced Drug Delivery Reviews. 2008;60(11):1278-88.

39. Vivero-Escoto JL, Slowing II, Trewyn BG, Lin VSY. Mesoporous silica nanoparticles for intracellular controlled drug delivery. Small. 2010;6(18):1952-67.

40. Trewyn BG, Giri S, Slowing II, Lin VSY. Mesoporous silica nanoparticle based controlled release, drug delivery and biosensor systems. Chemical Communications. 2007(31):3236-45.

41. Lin YS, Tsai CP, Huang HY, Kuo CT, Hung Y, Huang DM, et al. Well-ordered mesoporous silica nanoparticles as cell markers. Chemistry of Materials. 2005;17(18):4570-3

42. Lee CH, Lin TS, Mou CY. Mesoporous materials for encapsulating enzymes. Nano Today. 2009;4(2):165-79.

43. Kao KC, Lee CH, Lin TS, Mou CY. Cytochrome c covalently immobilized on mesoporous silicas as a peroxidase: Orientation effect. Journal of Materials Chemistry. 2010;20(22):4653-62.

44. Sun X, Trewyn BG. Porous Silica Nanoparticles for Drug Delivery and Controlled Release. Nanotechnology and Drug Delivery, Nanoplatforms in Drug Delivery. 2014;1:290.

45. Corma A. From microporous to mesoporous molecular sieve materials and their use in catalysis. Chemical Reviews. 1997;97(6):2373-420.

46. Bogush G, Tracy M, Zukoski C. Preparation of monodisperse silica particles: control of size and mass fraction. Journal of Non-crystalline Solids. 1988;104(1):95-106

47. Kresge C, Leonowicz M, Roth W, Vartuli J, Beck J. Ordered mesoporous molecular sieves synthesized by a liquid-crystal template mechanism. Nature. 1992;359(6397):710-2.

48. Zhao L, Qin H, Wu Ra, Zou H. Recent advances of mesoporous materials in sample preparation. Journal of Chromatography A. 2012;1228:193-204.

49. Chen CY, Burkett SL, Li HX, Davis ME. Studies on mesoporous materials II. Synthesis mechanism of MCM-41. Microporous Materials. 1993;2(1):27-34.

50. Xu W, Riikonen J, Lehto VP. Mesoporous systems for poorly soluble drugs. International Journal of Pharmaceutics. 2013;453(1):181-97.

51. Qu F, Zhu G, Lin H, Zhang W, Sun J, Li S, et al. A controlled release of ibuprofen by systematically tailoring the morphology of mesoporous silica materials. Journal of Solid State Chemistry. 2006;179(7):2027-35.

52. Kumar D, Chirravuri SS, Shastri NR. Impact of surface area of silica particles on dissolution rate and oral bioavailability of poorly water soluble drugs: a case study with aceclofenac. International Journal of Pharmaceutics. 2014;461(1):459-68.

53. Hu Y, Zhi Z, Zhao Q, Wu C, Zhao P, Jiang H, et al. 3D cubic mesoporous silica microsphere as a carrier for poorly soluble drug carvedilol. Microporous and Mesoporous Materials. 2012;147(1):94-101.

54. Kiekens F, Eelen S, Verheyden L, Daems T, Martens J, Den MGV. Use of ordered mesoporous silica to enhance the oral bioavailability of ezetimibe in dogs. Journal of Pharmaceutical Sciences. 2012;101(3):1136-44.

55. Speybroeck MV, Mellaerts R, Mols R, DoThi T, Martens JA, Humbeeck JV, et al. Enhanced absorption of the poorly soluble drug fenofibrate by tuning its release rate from ordered mesoporous silica. European Journal of Pharmaceutical Sciences. 2010;41(5):623-30.

56. Limnell T, Santos HA, Mäkilä E, Heikkilä T, Salonen J, Murzin DY, et al. Drug delivery formulations of ordered and nonordered mesoporous silica: comparison of three drug loading methods. Journal of Pharmaceutical Sciences. 2011;100(8):3294-306.

57. Speybroeck MV, Mellaerts R, Thi TD, Martens JA, Humbeeck JV, Annaert $\mathrm{P}$, et al. Preventing release in the acidic environment of the stomach via occlusion in ordered mesoporous silica enhances the absorption of poorly soluble weakly acidic drugs. Journal of Pharmaceutical Sciences. 2011;100(11):4864-76 
58. Xia X, Zhou C, Ballell L, Garcia-Bennett AE. In vivo Enhancement in Bioavailability of Atazanavir in the Presence of Proton-Pump Inhibitors using Mesoporous Materials. Chem Med Chem. 2012;7(1):43-8.

59. Wu J, Zhang L, Wang Y, Long Y, Gao H, Zhang X, et al. Mussel-inspired chemistry for robust and surface-modifiable multilayer films. Langmuir. 2011;27(22):13684-91.

60. Lee H, Dellatore SM, Miller WM, Messersmith PB. Mussel-inspired surface chemistry for multifunctional coatings. Science. 2007;318(5849):426-30.

61. Wiegemann M. Adhesion in blue mussels (Mytilus edulis) and barnacles (genus Balanus): mechanisms and technical applications. Aquatic SciencesResearch Across Boundaries. 2005;67(2):166-76.

62. Ding $\mathrm{Y}$, Floren M, Tan W. Mussel-inspired polydopamine for bio-surface functionalization. Biosurface and Biotribology. 2016;2(4):121-36.

63. Huang $\mathrm{Q}$, Liu M, Mao L, Xu D, Zeng $\mathrm{G}$, Huang $\mathrm{H}$, et al. Surface functionalized $\mathrm{SiO}_{2}$ nanoparticles with cationic polymers via the combination of mussel inspired chemistry and surface initiated atom transfer radical polymerization: Characterization and enhanced removal of organic dye. Journal of Colloid and Interface Science. 2017;499:170-9.

64. Zhang X, Wang S, Xu L, Feng L, Ji Y, Tao L, et al. Biocompatible polydopamine fluorescent organic nanoparticles: facile preparation and cell imaging. Nanoscale. 2012;4(18):5581-4.

65. Shi $\mathrm{Y}$, Jiang R, Liu M, Fu L, Zeng G, Wan Q, et al. Facile synthesis of polymeric fluorescent organic nanoparticles based on the self-polymerization of dopamine for biological imaging. Materials Science and Engineering: C. 2017;77:972-7.

66. Liu M, Zeng G, Wang K, Wan Q, Tao L, Zhang X, et al. Recent developments in polydopamine: an emerging soft matter for surface modification and biomedical applications. Nanoscale. 2016;8(38):16819-40.

67. Shi $Y$, Liu M, Deng F, Zeng G, Wan Q, Zhang X, et al. Recent progress and development on polymeric nanomaterials for photothermal therapy: a brief overview. Journal of Materials Chemistry B. 2017;5(2):194-206.

68. Zheng $\mathrm{X}$, Chen F, Zhang J, Cai K. Silica-assisted incorporation of polydopamine into the framework of porous nanocarriers by a facile one-pot synthesis. Journal of Materials Chemistry B. 2016;4(14):2435-43.

69. Song $Y$, Jiang $H$, Wang B, Kong $Y$, Chen J. Silver-incorporated musselinspired polydopamine coatings on mesoporous silica as an efficient nanocatalyst and antimicrobial agent. ACS Applied Materials and Interfaces. 2018;10(2):1792-801.

70. Wei Y, Gao L, Wang L, Shi L, Wei E, Zhou B, et al. Polydopamine and peptide decorated doxorubicin-loaded mesoporous silica nanoparticles as a targeted drug delivery system for bladder cancer therapy. Drug Delivery. 2017;24(1):681-91.

71. Giri S, Trewyn BG, Stellmaker MP, Lin VSY. Stimuli-responsive controlledrelease delivery system based on mesoporous silica nanorods capped with magnetic nanoparticles. Angewandte Chemie International Edition. 2005;44(32):5038-44.

72. Schlossbauer A, Warncke S, Gramlich PM, Kecht J, Manetto A, Carell T, et al. A Programmable DNA-Based Molecular Valve for Colloidal Mesoporous Silica. Angewandte Chemie International Edition. 2010;49(28):4734-7.

73. Schloßbauer A. Biofunctionalized mesoporous silica for controlled release applications: Imu. 2010.

74. LiZ, Barnes JC, Bosoy A, Stoddart JF, Zink JI. Mesoporous silica nanoparticles in biomedical applications. Chemical Society Reviews. 2012;41(7):2590-605.

75. Taylor-Pashow KM, Rocca JD, Lin W. Mesoporous silica nanoparticles with co-condensed gadolinium chelates for multimodal imaging. Nanomaterials. 2011;2(1):1-14.

76. Bergman L. Influence of surface functionalization on the behavior of silica nanoparticles in biological systems. 2014.

77. Lim MH, Stein A. Comparative studies of grafting and direct syntheses of inorganic-organic hybrid mesoporous materials. Chemistry of Materials. 1999;11(11):3285-95.

78. Liu R, Zhao X, Wu T, Feng P. Tunable redox-responsive hybrid nanogated ensembles. Journal of the American Chemical Society. 2008;130(44):144189.

79. She X, Chen L, Li C, He C, He L, Kong L. Functionalization of hollow mesoporous silica nanoparticles for improved 5-FU loading. Journal of Nanomaterials. 2015;16(1):108.
80. Zhu Y, Fang Y, Borchardt L, Kaskel S. PEGylated hollow mesoporous silica nanoparticles as potential drug delivery vehicles. Microporous and Mesoporous Materials. 2011;141(1):199-206.

81. Gulfam M, Chung BG. Development of $\mathrm{pH}$-responsive chitosan-coated mesoporous silica nanoparticles. Macromolecular Research. 2014;22(4):4127.

82. Senthilkumar R, Karaman DŞ, Paul P, Björk EM, Odén M, Eriksson JE, et al. Targeted delivery of a novel anticancer compound anisomelic acid using chitosan-coated porous silica nanorods for enhancing the apoptotic effect. Biomaterials Science. 2015;3(1):103-11.

83. Niemelä E, Desai D, Nkizinkiko Y, Eriksson JE, Rosenholm JM. Sugardecorated mesoporous silica nanoparticles as delivery vehicles for the poorly soluble drug celastrol enables targeted induction of apoptosis in cancer cells. European Journal of Pharmaceutics and Biopharmaceutics. 2015;96:11-21.

84. Yang KN, Zhang $\mathrm{CQ}$, Wang W, Wang PC, Zhou JP, Liang XJ. pH-responsive mesoporous silica nanoparticles employed in controlled drug delivery systems for cancer treatment. Cancer Biology and Medicine. 2014;11(1):3443.

85. Guha A, Biswas N, Bhattacharjee K, Das P, Kuotsu K. In vitro evaluation of $\mathrm{pH}$ responsive doxazosin loaded mesoporous silica nanoparticles: A smart approach in drug delivery. Current Drug Delivery. 2016;13(4):574-81.

86. Croissant JG, Qi C, Maynadier M, Cattoën X, Man MWC, Raehm L, et al. Multifunctional Gold-Mesoporous Silica Nanocomposites for Enhanced Two-Photon Imaging and Therapy of Cancer Cells. Frontiers in Molecular Biosciences. 2016;3:1.

87. Dobson J. Magnetic nanoparticles for drug delivery. Drug Development Research. 2006;67(1):55-60.

88. Liu Y, Zhang B, Yan B. Enabling anticancer therapeutics by nanoparticle carriers: the delivery of Paclitaxel. International Journal of Molecular Sciences. 2011;12(7):4395-413.

89. Yu M, Jambhrunkar S, Thorn P, Chen J, Gu W, Yu C. Hyaluronic acid modified mesoporous silica nanoparticles for targeted drug delivery to CD44overexpressing cancer cells. Nanoscale. 2013;5(1):178-83.

90. Su YL, Fang JH, Liao CY, Lin CT, Li YT, Hu H. Targeted Mesoporous Iron Oxide Nanoparticles-Encapsulated Perfluorohexane and a Hydrophobic Drug for Deep Tumor Penetration and Therapy. Theranostics. 2015;5(11):1233.

91. Grün M, Lauer I, Unger KK. The synthesis of micrometer-and submicrometersize spheres of ordered mesoporous oxide MCM-41. Advanced Materials. 1997;9(3):254-7.

92. Unger K, Kumar D, Grün M, Büchel G, Lüdtke S, Adam T, et al. Synthesis of spherical porous silicas in the micron and submicron size range: challenges and opportunities for miniaturized high-resolution chromatographic and electrokinetic separations. Journal of Chromatography A. 2000;892(1):47-55.

93. Huo Q, Feng J, Schüth F, Stucky GD. Preparation of hard mesoporous silica spheres. Chemistry of Materials. 1997;9(1):14-7.

94. Huh S, Wiench JW, Trewyn BG, Song S, Pruski M, Lin VSY. Tuning of particle morphology and pore properties in mesoporous silicas with multiple organic functional groups. Chemical Communications. 2003(18):2364-5.

95. Huh S, Wiench JW, Yoo JC, Pruski M, Lin VSY. Organic functionalization and morphology control of mesoporous silicas via a co-condensation synthesis method. Chemistry of materials. 2003;15(22):4247-56.

96. Cao QY, Jiang R, Liu M, Wan Q, Xu D, Tian J, et al. Microwave-assisted multicomponent reactions for rapid synthesis of AIE-active fluorescent polymeric nanoparticles by post-polymerization method. Materials Science and Engineering: C. 2017;80:578-83.

97. Luo J, Xie Z, Lam JW, Cheng L, Chen H, Qiu C, et al. Aggregationinduced emission of 1-methyl-1, 2, 3, 4, 5-pentaphenylsilole. Chemical Communications. 2001;(18):1740-1.

98. Mei J, Leung NL, Kwok RT, Lam JW, Tang BZ. Aggregation-induced emission: together we shine, united we soar!. Chemical Reviews. 2015;115(21):11718940.

99. Wang $\mathrm{K}$, Zhang $\mathrm{X}$, Zhang $\mathrm{X}$, Fan $\mathrm{X}$, Li Z, Huang Z, et al. Fabrication of photostable PEGylated polymer nanoparticles from AIE monomer and trimethylolpropane triacrylate. RSC Advances. 2015;5(92):75823-30.

100. Zhang X, Zhang X, Yang B, Liu M, Liu W, Chen Y, et al. Fabrication of aggregation induced emission dye-based fluorescent organic nanoparticles via emulsion polymerization and their cell imaging applications. Polymer Chemistry. 2014;5(2):399-404. 
101. Wan Q, Liu M, Xu D, Huang H, Mao L, Zeng G, et al. Facile fabrication of amphiphilic AIE active glucan via formation of dynamic bonds: self assembly, stimuli responsiveness and biological imaging. Journal of Materials Chemistry B. 2016;4(22):4033-9.

102. Liu M, Zhang X, Yang B, Deng F, Li Z, Wei J, et al. Water dispersible, non-cytotoxic, cross-linked luminescent AIE dots: Facile preparation and bioimaging applications. Applied Surface Science. 2014;322:155-61.

103. Jiang R, Liu M, Chen T, Huang H, Huang Q, Tian J, et al. Facile construction and biological imaging of cross-linked fluorescent organic nanoparticles with aggregation-induced emission feature through a catalyst-free azide-alkyne click reaction. Dyes and Pigments. 2018;148:52-60.

104. Cao QY, Jiang R, Liu M, Wan Q, Xu D, Tian J, et al. Preparation of AIE-active fluorescent polymeric nanoparticles through a catalyst-free thiol-yne click reaction for bioimaging applications. Materials Science and Engineering: C. 2017;80:411-6.

105. Huang $\mathrm{H}$, Liu $\mathrm{M}$, Wan $\mathrm{Q}$, Jiang $\mathrm{R}$, Xu D, Huang $\mathrm{Q}$, et al. Facile fabrication of luminescent hyaluronic acid with aggregation-induced emission through formation of dynamic bonds and their theranostic applications. Materials Science and Engineering: C. 2018;91:201-7.

106. Jiang $\mathrm{R}$, Liu $\mathrm{H}$, Liu M, Tian J, Huang $\mathrm{Q}$, Huang $\mathrm{H}$, et al. A facile one-pot Mannich reaction for the construction of fluorescent polymeric nanoparticles with aggregation-induced emission feature and their biological imaging. Materials Science and Engineering: C. 2017;81:416-21.

107. Jiang R, Liu M, Li C, Huang Q, Huang H, Wan Q, et al. Facile fabrication of luminescent polymeric nanoparticles containing dynamic linkages via a onepot multicomponent reaction: Synthesis, aggregation-induced emission and biological imaging. Materials Science and Engineering: C. 2017;80:708-14.

108. Huang $H, X u$ D, Liu M, Jiang R, Mao L, Huang $Q$, et al. Direct encapsulation of AIE-active dye with $\beta$ cyclodextrin terminated polymers: Self-assembly and biological imaging. Materials Science and Engineering: C. 2017;78:862-7.

109. Zhao S, Xu M, Cao C, Yu Q, Zhou Y, Liu J. A redox-responsive strategy using mesoporous silica nanoparticles for co-delivery of siRNA and doxorubicin. Journal of Materials Chemistry B. 2017.

110. Lin YS, Wu SH, Hung Y, Chou YH, Chang C, Lin ML, et al. Multifunctional composite nanoparticles: magnetic, luminescent and mesoporous. Chemistry of Materials. 2006;18(22):5170-2.

111. Chen H, Zhen Z, Tang W, Todd T, Chuang YJ, Wang L, et al. Label-free luminescent mesoporous silica nanoparticles for imaging and drug delivery. Theranostics. 2013;3(9):650.

112. Hu X, Gao X. Silica- Polymer Dual Layer-Encapsulated Quantum Dots with Remarkable Stability. Acs Nano. 2010;4(10):6080-6.

113. Idris NM, Gnanasammandhan MK, Zhang J, Ho PC, Mahendran R, Zhang Y. In vivo photodynamic therapy using upconversion nanoparticles as remotecontrolled nanotransducers. Nature Medicine. 2012;18(10):1580.

114. Qian HS, Guo HC, Ho PCL, Mahendran R, Zhang Y. Mesoporous-Silicacoated up-conversion fluorescent nanoparticles for photodynamic therapy. Small. 2009;5(20):2285-90.

115. Wang L, Wang K, Santra S, Zhao X, Hilliard LR, Smith JE, et al. Watching silica nanoparticles glow in the biological world. ACS Publications. 2006.

116. Auger A, Samuel J, Poncelet O, Raccurt O. A comparative study of noncovalent encapsulation methods for organic dyes into silica nanoparticles. Nanoscale Research Letters. 2011;6(1):328.

117. Tasciotti E, Liu X, Bhavane R, Plant K, Leonard AD, Price BK, et al. Mesoporous silicon particles as a multistage delivery system for imaging and therapeutic applications. Nature Nanotechnology. 2008;3(3):151.

118. Park JH, Gu L, Maltzahn GV, Ruoslahti E, Bhatia SN, Sailor MJ. Biodegradable luminescent porous silicon nanoparticles for in vivo applications. Nature Materials. 2009;8(4):331.

119. Jain B, Reeja K, Mondal P, Sinha AK. Luminescent mesoporous silica nanoparticles for biomedical applications: Synthesis and characterization. Journal of Luminescence. 2018;200:200-5.

120. Zhang X, Wang K, Liu M, Zhang X, Tao L, Chen Y, et al. Polymeric AlE-based nanoprobes for biomedical applications: recent advances and perspectives. Nanoscale. 2015;7(27):11486-508.

121. Long Z, Liu M, Jiang R, Wan Q, Mao L, Wan $Y$, et al. Preparation of water soluble and biocompatible AIE-active fluorescent organic nanoparticles via multicomponent reaction and their biological imaging capability. Chemical Engineering Journal. 2017;308:527-34.
122. Long Z, Liu M, Wang K, Deng F, Xu D, Liu L, et al. Facile synthesis of AIE-active amphiphilic polymers: Self-assembly and biological imaging applications. Materials Science and Engineering: C. 2016;66:215-20.

123. Long Z, Mao L, Liu M, Wan Q, Wan Y, Zhang X, et al. Marrying multicomponent reactions and aggregation-induced emission (AIE): new directions for fluorescent nanoprobes. Polymer Chemistry. 2017;8(37):5644-54.

124. Huang L, Yang S, Chen J, Tian J, Huang Q, Huang H, et al. A facile surface modification strategy for fabrication of fluorescent silica nanoparticles with the aggregation-induced emission dye through surface-initiated cationic ring opening polymerization. Materials Science and Engineering: C. 2019;94:2708.

125. Zhang X, Zhang X, Yang B, Liu M, Liu W, Chen Y, et al. Polymerizable aggregation-induced emission dye-based fluorescent nanoparticles for cell imaging applications. Polymer Chemistry. 2014;5(2):356-60.

126. Jiang R, Liu M, Huang H, Mao L, Huang Q, Wen $\mathrm{Y}$, et al. Facile fabrication of organic dyed polymer nanoparticles with aggregation-induced emission using an ultrasound-assisted multicomponent reaction and their biological imaging. Journal of Colloid and Interface Science. 2018;519:137-44.

127. Aznar E, Marcos MD, Martínez-Máñez RN, Sancenón F, Soto J, Amorós $P$, et al. $\mathrm{pH}$-and photo-switched release of guest molecules from mesoporous silica supports. Journal of the American Chemical Society. 2009;131(19):6833-43.

128. Luo Z, Ding $X, H u Y$, Wu S, Xiang $Y$, Zeng $Y$, et al. Engineering a hollow nanocontainer platform with multifunctional molecular machines for tumortargeted therapy in vitro and in vivo. ACS Nano. 2013;7(11):10271-84.

129. Ketkar-Atre A, Struys T, Dresselaers T, Hodenius M, Mannaerts I, Ni Y, et al. In vivo hepatocyte MR imaging using lactose functionalized magnetoliposomes. Biomaterials. 2014;35(3):1015-24.

130. Morlieras J, Dufort S, Sancey L, Truillet C, Mignot A, Rossetti F, et al. Functionalization of small rigid platforms with cyclic RGD peptides for targeting tumors overexpressing av $\beta 3$-integrins. Bioconjugate Chemistry. 2013;24(9):1584-97.

131. Geninatti CS, Alberti D, Szabo I, Aime S, Djanashvili K. MRI Visualization of Melanoma Cells by Targeting Overexpressed Sialic Acid with a GdIIIdota-en-pba Imaging Reporter. Angewandte Chemie International Edition. 2013;52(4):1161-4.

132. Thomas CR, Ferris DP, Lee JH, Choi E, Cho MH, Kim ES, et al. Noninvasive remote-controlled release of drug molecules in vitro using magnetic actuation of mechanized nanoparticles. Journal of the American Chemical Society. 2010;132(31):10623-5.

133. Sun YL, Zhou Y, Li QL, Yang YW. Enzyme-responsive supramolecular nanovalves crafted by mesoporous silica nanoparticles and cholinesulfonatocalix [4] arene [2] pseudorotaxanes for controlled cargo release. Chemical Communications. 2013;49(79):9033-5.

134. Patel K, Angelos S, Dichtel WR, Coskun A, Yang YW, Zink JI, et al. Enzymeresponsive snap-top covered silica nanocontainers. Journal of the American Chemical Society. 2008;130(8):2382-3.

135. Sun Y, Sai H, Spoth KA, Tan KW, Werner-Zwanziger U, Zwanziger J, et al. Stimuli-responsive shapeshifting mesoporous silica nanoparticles. Nano Letters. 2015;16(1):651-5.

136. Moreira AF, Dias DR, Correia IJ. Stimuli-responsive mesoporous silica nanoparticles for cancer therapy: A review. Microporous and Mesoporous Materials. 2016;236:141-57.

137. Liu J, Luo Z, Zhang J, Luo T, Zhou J, Zhao X, et al. Hollow mesoporous silica nanoparticles facilitated drug delivery via cascade $\mathrm{pH}$ stimuli in tumor microenvironment for tumor therapy. Biomaterials. 2016;83:51-65.

138. Hakeem A, Zahid F, Duan R, Asif M, Zhang T, Zhang Z, et al. Cellulose conjugated FITC-labelled mesoporous silica nanoparticles: intracellular accumulation and stimuli responsive doxorubicin release. Nanoscale. 2016;8(9):5089-97.

139. Chen X, Liu Z, Parker SG, Zhang X, Gooding JJ, Ru Y, et al. Light-induced hydrogel based on tumor-targeting mesoporous silica nanoparticles as a theranostic platform for sustained cancer treatment. ACS Applied Materials and Interfaces. 2016;8(25):15857-63.

140. Guo X, Guo N, Zhao J, Cai Y. Active targeting co-delivery system based on hollow mesoporous silica nanoparticles for antitumor therapy in ovarian cancer stem-like cells. Oncology Reports. 2017;38(3):1442-50. 
141. Wen J, Yang K, Xu Y, Li H, Liu F, Sun S. Construction of A Triple-StimuliResponsive System Based on Cerium Oxide Coated Mesoporous Silica Nanoparticles. Scientific Reports. 2016;6:38931.

142. Cho IH, Shim MK, Jung B, Jang EH, Park MJ, Kang HC, et al. Heat shock responsive drug delivery system based on mesoporous silica nanoparticles coated with temperature sensitive gatekeeper. Microporous and Mesoporous Materials. 2017;253:96-101.

143. Cheng W, Nie J, Xu L, Liang C, Peng Y, Liu G, et al. A pH-sensitive delivery vehicle based on folic acid-conjugated polydopamine-modified mesoporous silica nanoparticles for targeted cancer therapy. ACS Applied Materials and Interfaces. 2017.

144. Sun L, Wang D, Chen Y, Wang L, Huang P, Li Y, et al. Core-shell hierarchical mesostructured silica nanoparticles for gene/chemo-synergetic stepwise therapy of multidrug-resistant cancer. Biomaterials. 2017;133:219-28.

145. Li T, Chen X, Liu Y, Fan L, Lin L, Xu Y, et al. pH-Sensitive mesoporous silica nanoparticles anticancer prodrugs for sustained release of ursolic acid and the enhanced anti-cancer efficacy for hepatocellular carcinoma cancer. European Journal of Pharmaceutical Sciences. 2017;96:456-63.

146. Kumar B, Kulanthaivel S, Mondal A, Mishra S, Banerjee B, Bhaumik A, et al. Mesoporous silica nanoparticle based enzyme responsive system for colon specific drug delivery through guar gum capping. Colloids and Surfaces B: Biointerfaces. 2017;150:352-61.

147. Hanahan D, Weinberg RA. Hallmarks of cancer: the next generation. Cell. 2011;144(5):646-74.

148. Ahmadi NN, Hassani KH, Beygzadeh M, Teimourian S, Kazemzad M. Delivery of curcumin by a $\mathrm{pH}$-responsive chitosan mesoporous silica nanoparticles for cancer treatment. Artificial Cells, Nanomedicine and Biotechnology. 2017;17.

149. Kienzle A, Kurch S, Schlöder J, Berges C, Ose R, Schupp J, et al. Dendritic Mesoporous Silica Nanoparticles for pH-Stimuli-Responsive Drug Delivery of TNF-Alpha. Advanced Healthcare Materials. 2017.

150. Zhou S, Wu D, Yin X, Jin X, Zhang $X$, Zheng $S$, et al. Intracellular pHresponsive and rituximab-conjugated mesoporous silica nanoparticles for targeted drug delivery to lymphoma B cells. Journal of Experimental and Clinical Cancer Research. 2017;36(1):24.

151. Lumeng JY, Wall BA, Wangari-Talbot J, Chen S. Metabotropic glutamate receptors in cancer. Neuropharmacology. 2017;115:193-202.

152. Chen X, Sun H, Hu J, Han X, Liu H, Hu Y. Transferrin gated mesoporous silica nanoparticles for redox-responsive and targeted drug delivery. Colloids and Surfaces B: Biointerfaces. 2017;152:77-84.

153. Kang T, Li F, Baik S, Shao W, Ling D, Hyeon T. Surface design of magnetic nanoparticles for stimuli-responsive cancer imaging and therapy. Biomaterials. 2017;136:98-114.

154. Hauser AK, Wydra RJ, Stocke NA, Anderson KW, Hilt JZ. Magnetic nanoparticles and nanocomposites for remote controlled therapies. Journal of Controlled Release. 2015;219:76-94.

155. Li T, Shen X, Zhang C, Yang H, Wu C, Liu Y. Magnetic mesoporous silica nanoparticles co-delivering doxorubicin and VEGF siRNA for cervical cancer targeting therapy and MR imaging. Nanomedicine: Nanotechnology, Biology and Medicine. 2016;12(2):521.

156. Kim J, Cho HR, Jeon H, Kim D, Song C, Lee N, et al. Continuous $\mathrm{O}_{2}$-Evolving $\mathrm{MnFe}_{2} \mathrm{O}_{4}$ Nanoparticle-Anchored Mesoporous Silica Nanoparticles for Efficient Photodynamic Therapy in Hypoxic Cancer. Journal of the American Chemical Society. 2017;139(32):10992-5.

157. Tian Z, Yu X, Ruan Z, Zhu M, Zhu Y, Hanagata N. Magnetic mesoporous silica nanoparticles coated with thermo-responsive copolymer for potential chemo-and magnetic hyperthermia therapy. Microporous and Mesoporous Materials. 2018;256:1-9.

158. Hu C, Huang P, Zheng Z, Yang Z, Wang X. A Facile Strategy to Prepare an Enzyme-Responsive Mussel Mimetic Coating for Drug Delivery Based on Mesoporous Silica Nanoparticles. Langmuir. 2017;33(22):5511-8.

159. Tukappa A, Ultimo A, Dela TPC, Vicente MTP, Galarza FS, Martínez-Máñez R. Polyglutamic acid-gated mesoporous silica nanoparticles for enzymecontrolled drug delivery. Langmuir; 2016. American Chemical Society.

160. Lu J, Choi E, Tamanoi F, Zink Jl. Light-Activated Nanoimpeller-Controlled Drug Release in Cancer Cells. Small. 2008;4(4):421-6.
161. Fu Q, Rao GR, Ista LK, Wu Y, Andrzejewski BP, Sklar LA, et al. Control of molecular transport through stimuli-responsive ordered mesoporous materials. Advanced Materials. 2003;15(15):1262-6.

162. Aznar E, Mondragón L, Ros-Lis JV, Sancenón F, Marcos MD, MartínezMáñez R, et al. Finely tuned temperature-controlled cargo release using paraffin-capped mesoporous silica nanoparticles. Angewandte Chemie International Edition. 2011;50(47):11172-5.

163. Wang Y, Cui Y, Zhao Y, He B, Shi X, Di D, et al. Fluorescent carbon dotgated multifunctional mesoporous silica nanocarriers for redox/enzyme dualresponsive targeted and controlled drug delivery and real-time bioimaging. European Journal of Pharmaceutics and Biopharmaceutics. 2017;117:10515.

164. Zhu S, Zhou Z, Zhang D, Jin C, Li Z. Design and synthesis of delivery system based on SBA-15 with magnetic particles formed in situ and thermo-sensitive PNIPA as controlled switch. Microporous and Mesoporous Materials. 2007;106(1):56-61.

165. Khosravian P, Ardestani MS, Khoobi M, Ostad SN, Dorkoosh FA, Javar $\mathrm{HA}$, et al. Mesoporous silica nanoparticles functionalized with folic acid/ methionine for active targeted delivery of docetaxel. OncoTargets and Therapy. 2016;9:7315.

166. Goel S, Chen F, Hong H, Valdovinos H, Barnhart T, Cai W. VEGFR-targeted drug delivery in vivo with mesoporous silica nanoparticles. Journal of Nuclear Medicine. 2014;55(supplement 1):222.

167. Saroj S, Rajput SJ. Composite smart mesoporous silica nanoparticles as promising theranostic candidates: Recent trends and applications. Journal of Drug Delivery Science and Technology. 2018.

168. Lu F, Wu SH, Hung Y, Mou CY. Size effect on cell uptake in well-suspended, uniform mesoporous silica nanoparticles. Small. 2009;5(12):1408-13.

169. He Q, Zhang Z, Gao Y, Shi J, Li Y. Intracellular localization and cytotoxicity of spherical mesoporous silica nano-and microparticles. Small. 2009;5(23):2722-9.

170. Lin YS, Haynes CL. Impacts of mesoporous silica nanoparticle size, pore ordering and pore integrity on hemolytic activity. Journal of the American Chemical Society. 2010;132(13):4834-42.

171. Chen G, Teng Z, Su X, Liu Y, Lu G. Unique biological degradation behavior of Stöber mesoporous silica nanoparticles from their interiors to their exteriors. Journal of Biomedical Nanotechnology. 2015;11(4):722-9.

172. Zhai W, He C, Wu L, Zhou Y, Chen H, Chang J, et al. Degradation of hollow mesoporous silica nanoparticles in human umbilical vein endothelial cells. Journal of Biomedical Materials Research Part B: Applied Biomaterials. 2012;100(5):1397-403.

173. Hanafi-Bojd MY, Moosavian KSA, Taghdisi SM, Ansari L, Abnous K, Malaekeh-Nikouei B. MUC1 Aptamer-conjugated mesoporous silica nanoparticles effectively target breast cancer cells. Drug Development and Industrial Pharmacy. 2017;1-6.

174. Ma'mani L, Nikzad S, Kheiri-Manjili H, al-Musawi S, Saeedi M, Askarlou S, et al. Curcumin-loaded guanidine functionalized PEGylated I3ad mesoporous silica nanoparticles KIT-6: Practical strategy for the breast cancer therapy. European Journal of Medicinal Chemistry. 2014;83:646-54.

175. Song H, Su C, Cui W, Zhu B, Liu L, Chen Z, et al. Folic acid-chitosan conjugated nanoparticles for improving tumor-targeted drug delivery. BioMed Research International. 2013;2013.

176. Wang T, Liu Y, Wu C. Effect of paclitaxel-mesoporous silica nanoparticles with a core-shell structure on the human lung cancer cell line A549. Nanoscale Research Letters. 2017;12(1):66.

177. Gao Y, Che X, Zheng C, Hou K, Qu X, Liu Y, et al. Effect of an AlbuminCoated Mesoporous Silicon Nanoparticle Platform for Paclitaxel Delivery in Human Lung Cancer Cell Line A549. Journal of Nanomaterials. 2016;2016.

178. Sundarraj S, Thangam R, Sujitha MV, Vimala K, Kannan S. Ligandconjugated mesoporous silica nanorattles based on enzyme targeted prodrug delivery system for effective lung cancer therapy. Toxicology and Applied Pharmacology. 2014;275(3):232-43.

179. Chaix A, ElCheikh K, Bouffard E, Maynadier M, Aggad D, Stojanovic V, et al. Mesoporous silicon nanoparticles for targeted two-photon theranostics of prostate cancer. Journal of Materials Chemistry B. 2016;4(21):3639-42.

180. Xie X, Li F, Zhang H, Lu Y, Lian S, Lin H, et al. EpCAM aptamer-functionalized mesoporous silica nanoparticles for efficient colon cancer cell-targeted drug delivery. European Journal of Pharmaceutical Sciences. 2016;83:28-35. 
181. She X, Chen L, Velleman L, Li C, Zhu H, He C, et al. Fabrication of high specificity hollow mesoporous silica nanoparticles assisted by Eudragit for targeted drug delivery. Journal of Colloid and Interface Science. 2015;445:151-60.

182. Tan L, Yang MY, Wu HX, Tang ZW, Xiao JY, Liu CJ, et al. Glucose-and pHResponsive Nanogated Ensemble Based on Polymeric Network Capped Mesoporous Silica. ACS Applied Materials and Interfaces. 2015;7(11):6310-6.

183. Wang Z, Wu P, He Z, He H, Rong W, Li J, et al. Mesoporous silica nanoparticles with lactose-mediated targeting effect to deliver platinum (iv) prodrug for liver cancer therapy. Journal of Materials Chemistry B. 2017;5(36):7591-7.

184. Liu X, Situ A, Kang Y, Villabroza KR, Liao $\mathrm{Y}$, Chang $\mathrm{CH}$, et al. Irinotecan delivery by lipid-coated mesoporous silica nanoparticles shows improved efficacy and safety over liposomes for pancreatic cancer. ACS Nano. 2016;10(2):2702-15.

185. Zhang Y, Guo J, Zhang XL, Li DP, Zhang TT, Gao FF, et al. Antibody fragment-armed mesoporous silica nanoparticles for the targeted delivery of bevacizumab in ovarian cancer cells. International Journal of Pharmaceutics. 2015;496(2):1026-33.

186. Jia L, Li Z, Shen J, Zheng D, Tian X, Guo H, et al. Multifunctional mesoporous silica nanoparticles mediated co-delivery of paclitaxel and tetrandrine for overcoming multidrug resistance. International Journal of Pharmaceutics. 2015;489(1):318-30.

187. Meng $\mathrm{H}$, Mai WX, Zhang $\mathrm{H}$, Xue $\mathrm{M}$, Xia T, Lin S, et al. Codelivery of an optimal drug/siRNA combination using mesoporous silica nanoparticles to overcome drug resistance in breast cancer in vitro and in vivo. ACS Nano. 2013;7(2):994-1005

188. Cheng SH, Lee CH, Chen MC, Souris JS, Tseng FG, Yang CS, et al. Trifunctionalization of mesoporous silica nanoparticles for comprehensive cancer theranostics- the trio of imaging, targeting and therapy. Journal of Materials Chemistry. 2010;20(29):6149-57.

189. Gurka MK, Pender D, Chuong P, Fouts BL, Sobelov A, McNally MW, et al. Identification of pancreatic tumors in vivo with ligand-targeted, $\mathrm{pH}$ responsive mesoporous silica nanoparticles by multispectral optoacoustic tomography. Journal of Controlled Release. 2016;231:60-7.
190. Nagesetti A, Srinivasan S, McGoron AJ. Polyethylene glycol modified ORMOSIL theranostic nanoparticles for triggered doxorubicin release and deep drug delivery into ovarian cancer spheroids. Journal of Photochemistry and Photobiology B: Biology. 2017;174:209-16.

191. Chen H, Sun X, Wang GD, Nagata K, Hao Z, Wang A, et al. LiGa 5 O 8: Cr-based theranostic nanoparticles for imaging-guided $X$-ray induced photodynamic therapy of deep-seated tumors. Materials Horizons. 2017.

192. Hsiao SM, Peng BY, Tseng YS, Liu HT, Chen CH, Lin HM. Preparation and characterization of multifunctional mesoporous silica nanoparticles for dual magnetic resonance and fluorescence imaging in targeted cancer therapy. Microporous and Mesoporous Materials. 2017.

193. Cheng B, He H, Huang T, Berr SS, He J, Fan D, et al. Gold nanosphere gated mesoporous silica nanoparticle responsive to near-infrared light and redox potential as a theranostic platform for cancer therapy. Journal of biomedical nanotechnology. 2016;12(3):435-49.

194. Prasad R, Aiyer S, Chauhan DS, Srivastava R, Selvaraj K. Bioresponsive carbon nano-gated multifunctional mesoporous silica for cancer theranostics. Nanoscale. 2016;8(8):4537-46.

195. Babaei M, Abnous K, Taghdisi SM, Amel Farzad S, Peivandi MT, Ramezani $\mathrm{M}$, et al. Synthesis of theranostic epithelial cell adhesion molecule targeted mesoporous silica nanoparticle with gold gatekeeper for hepatocellular carcinoma. Nanomedicine. 2017;12(11):1261-69.

196. Feng L, Gai S, He F, Dai Y, Zhong C, Yang P, et al. Multifunctional mesoporous $\mathrm{ZrO}_{2}$ encapsulated upconversion nanoparticles for mild NIR light activated synergistic cancer therapy. Biomaterials. 2017;147:39-52.

197. Lin CY, Li WP, Huang SP, Yeh CS, Yang CM. Hollow mesoporous silica nanosphere-supported FePt nanoparticles for potential theranostic applications. Journal of Materials Chemistry B. 2017;5(36):7598-607.

198. Shi Y, Miller ML, Di Pasqua AJ. Biocompatibility of mesoporous silica nanoparticles?. Comments on Inorganic Chemistry. 2016;36(2):61-80.

199. Phillips E, Penate-Medina O, Zanzonico PB, Carvajal RD, Mohan P, Ye $\mathrm{Y}$, et al. Clinical translation of an ultrasmall inorganic optical-PET imaging nanoparticle probe. Science Translational Medicine. 2014;6(260):260ra149.s

\section{PICTORIAL ABSTRACT}

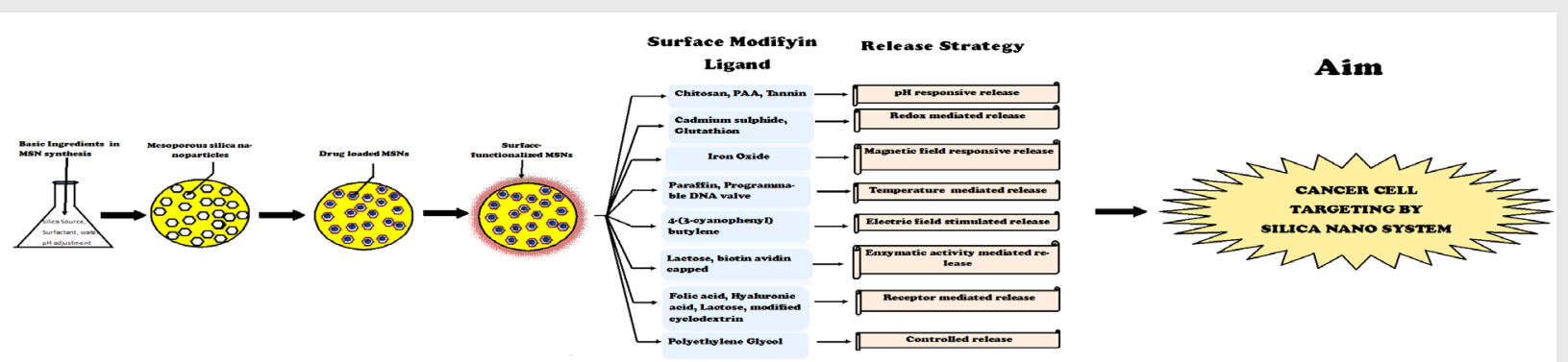

\section{SUMMARY}

In this review, we have emphasized on the utility of novel approaches of multifunctional mesoporous silica nanoparticles. Property of cell specificity helps in targeting the MSNs to tumor cells by modifying the silanol group presented on external surface. A tailor-made approach of MSNs gives an option of tuning the particle size and particle shape as per the user requirement. Biocompatibility investigation of MSNs has demonstrated its safe nature. Despite of having numerous advantages, an in-depth research is still needed to be done on MSNs to make the pharmaceutical world more familiar with MSNs applications.

Cite this article: Shah PV, Rajput S. Surface Decorated Mesoporous Silica Nanoparticles: A Promising and Emerging Tool for Cancer Targeting. Indian J of Pharmaceutical Education and Research. 2019;53(3):382-99. 